Copyright (C) 2015 IEEE. Personal use of this material is permitted. Permission from IEEE must be obtained for all other uses, in any current or future media, including reprinting/republishing this material for advertising or promotional purposes, creating new collective works, for resale or redistribution to servers or lists, or reuse of any copyrighted component of this work in other works. 


\title{
MSE-Based Transceiver Designs for Full-Duplex MIMO Cognitive Radios
}

\author{
Ali Cagatay Cirik, Member, IEEE, Rui Wang, Member, IEEE, Yue Rong, Senior Member, IEEE, and Yingbo Hua, \\ Fellow, IEEE
}

\begin{abstract}
We study two scenarios of full duplex (FD) multipleinput multiple-output (MIMO) cognitive radio networks: FD cognitive ad-hoc networks and FD cognitive cellular networks. In FD cognitive ad-hoc networks (also referred as interference channels), each pair of secondary users (SUs) operate in FD mode and communicate with each other within the service range of primary users (PUs). Each SU experiences not only self- interference, but also inter-user interference from all other SUs, and all SUs generate interference on PUs. We address two optimization problems: one is to minimize the sum of mean-squared- errors (MSE) of all estimated symbols, and the other is to minimize the maximum per-SU MSE of estimated symbols, both of which are subject to power constraints at SUs and interference constraints projected to each PU. We show that these problems can be cast as a second-order cone programming (SOCP), and joint design of transceiver matrices can be obtained through an iterative algorithm. Moreover, we show that the proposed algorithm is not only applicable to interference channels, but also applicable to FD cellular systems, in which a base station operating in FD mode simultaneously serves multiple uplink and downlink users, and it is shown to outperform HD scheme significantly.
\end{abstract}

Index Terms-Cognitive radio, full-duplex, interference channels, MIMO, MSE, multi-user, self-interference, transceiver designs.

\section{INTRODUCTION}

$\mathbf{T}$ $\mathrm{HE}$ increasing demand for the improved spectral efficiency with the proliferation of wireless services is calling for powerful communication technologies that deliver increasing data rates and utilize the current spectrum resources more efficiently. In half-duplex (HD) wireless communication systems, namely time-division duplex (TDD) and frequencydivision duplex (FDD), a node can either transmit or receive on a single frequency band, but not simultaneously. This incurs significant loss of spectrum efficiency. With full-duplex (FD) communication system, a node receives and transmits simultaneously on the same channel [1]-[29], which can potentially double its link capacity, and increase its spectral efficiency targeted by the next generation wireless communication systems.

The research leading to these results has received funding in part by Army Research Office under grant no. W911NF1210432 and University of California Office of the President under Grant no. PC-12-247260, in part from the Academy of Finland under Grant 260755 - Project JULIET.

A. C. Cirik is with Centre for Wireless Communications, University of Oulu, Oulu, 90570, Finland (email: acirik@ee.oulu.fi).

R. Wang is with the Department of Electronics and Information Engineering, Tongji University, Shanghai, 201804, P. R. China (email: ruiwang@tongji.edu.cn).

Y. Rong is with the Department of Electrical and Computer Engineering, Curtin University, Bentley, WA 6102, Australia (email: y.rong@curtin.edu.au).

Y. Hua is with the Department of Electrical Engineering, University of California, Riverside, CA 92521, USA (email: yhua@ee.ucr.edu).
There has been a recent growth of interest in the high spectral efficiency gain of a FD radio over a HD radio. In particular, FD relaying has drawn much interest since it is effective in extending the network coverage and improving the link reliability of the network [1], [10], [11]. In addition to relay nodes, small cells which extend the service coverage and/or increase network capacity can be deployed in the FD mode due to low transmit powers, short transmission distances and low mobility. A small cell network where a FD base station (BS) serves multiple uplink (UL) and downlink (DL) users operating in the HD mode has been considered in [2][5]. Moreover, cognitive radios, which substantially increase spectrum utilization efficiency by allowing unlicensed users to share the spectrum with licensed users, can be deployed in the FD mode. A FD cognitive radio can transmit and sense the transmission status of other nodes [6], [7]. FD technology is suitable to combat several problems at the medium access control (MAC) layer, such as hidden terminals, large delays, and congestion [8]-[9].

The limiting factor on practical implementation of FD radios is the so-called self-interference at the front-end of the receiver, which is caused by the signal leakage from the transmitter antennas of a FD node to its own receiver antennas. The power of the self-interference signal can be $100 \mathrm{~dB}$ stronger than the received signal of interest coming from a distant source, which can exceed the dynamic range of the analog-to-digital converter (ADC). Unless this selfinterference is canceled satisfactorily, a radio transceiver cannot perform FD operation. Recently several research groups have developed methods for self-interference cancellation. These works include the transmit beamforming (spatial domain suppression) methods in [10]-[12], in which the selfinterference is canceled at the front-end of the receiver by a cancellation signal generated from a transmitted signal in the baseband. Promising results from experimental research that demonstrate the feasibility of FD transmission using the offthe-shelf hardware are also available in [9], [13]-[16]. However, due to imperfect self-interference channel knowledge and the hardware impairment in the transmitter chain (amplifier non-linearity, phase noise, and I/Q channel imbalance), the self-interference cannot be canceled completely in practice. Therefore, further optimization of a FD radio, such as power allocation and beamformer design, must take into account the residual self-interference [17]-[26]. In this paper, we refer to the above optimization problems as transceiver design.

Cognitive radio system is also a promising technology to enhance spectrum efficiency [30], [31]. In cognitive radio 
systems, a set of unlicensed secondary users (SUs) operate within the service range of licensed primary users (PUs) where the amount of interference from SUs to PUs must be constrained to meet the Quality-of-Service (QoS) requirements for the PUs [32]-[45].

Examples of transceiver design for a single pair of FD radio nodes are available in [12], [17]-[26]. But there has been little work on transceiver design for multiple pairs of FD radio nodes. Recently, the interest on MIMO channels has migrated from point-to-point MIMO and MIMO downlink channel to MIMO interference channels because the latter is inherent in many practical problems [46]. With the increase of wireless devices that share the same frequency and time resources, interference becomes the key bottleneck that limits the performance of communication networks. Studies on the performance of cellular communication systems where each cell causes interference to other cells can be carried out by focusing on MIMO interference channels [47]. In this paper, we propose a joint and iterative transceiver design method for a MIMO FD cognitive interference channel (i.e., an ad-hoc FD cognitive network) and a FD cognitive cellular system by taking into account the limited dynamic ranges of the transmitter and receivers.

Particularly, we consider $K$ pairs of FD SUs exchanging information, while the SUs also provide protection to multiple PUs. The nodes in each SU pair suffer not only from selfinterference due to operating in the FD mode, but also from inter-user interference due to simultaneous transmission from all SUs. We first consider the sum mean-squared-error (SumMSE) as the objective function to minimize subjecting to power constraints at the SUs and interfering power constraints at the PUs. An iterative algorithm which optimizes the transmit and receiving beamforming matrices in alternating manner is proposed. At each iteration, Sum-MSE decreases monotonically, and is guaranteed to converge to at least a local optimum solution. However, in a multiuser MIMO systems, with an optimization of overall efficiency, the transmission power resource is focused on the good channels, i.e., the good channels are favored over the bad channels, where some users are not even allowed to transmit their signals. In order to avoid this effect, we also consider the problem of minimizing the maximum per-node MSE (Min-Max) subject to power constraints at the SUs and interfering power constraints at the PUs. It is shown in the simulations that while the proposed Sum-MSE minimization scheme achieves smaller total MSE, the proposed Min-Max scheme achieves the same MSE for every user.

Moreover, we show that the proposed algorithm can also be applied to FD cognitive cellular systems. In current cellular systems, DL and UL channels operate either in orthogonal time or frequency domain, resulting in inefficient use of the radio resources. In this paper, we consider a scenario where a BS operating in FD mode communicates with UL and DL users operating in HD mode simultaneously. In addition to self-interference at the BS, the optimization problem is exacerbated by the co-channel interference (CCI) caused by the UL users to DL users. Sum-rate maximization for FD multi-user systems has been investigated in [2], [27], [28], [29]. However, the CCI is not taken into account in [27], single-antenna users are assumed in [2], and these works [2], [27] and [28] did not consider any transmitter/receiver distortion. In this paper, we take into account the major hardware impairments in practical transceivers. The simulation results show that the proposed FD system can achieve a significant improvement of throughput over HD system.

\section{A. Rationale for MSE-Based Optimizations}

MSE-based transceiver designs have been considered extensively due to its good performance and significantly reduced complexity. It has been shown in [48] that minimum meansquared-error (MMSE) estimation plays an important role in approaching the information-theoretic limits of Gaussian channels. When MMSE receiver is used, MSE-based optimization problems are equivalent to signal-to-interference-plusnoise ratio (SINR)-based optimization problems, since they are related as [49],

$$
\mathrm{MSE}=\frac{1}{1+\mathrm{SINR}} .
$$

Therefore, rate-based optimization using $\log _{2}(1+$ SINR $)$ can be conveniently transformed into MSE-based optimization, $-\log _{2}$ (MSE). And as mentioned in [50], the user-wise MSE can be used to approximate the achievable rate of the users when they jointly decode their streams. In particular, when MMSE receivers are employed, the achievable rate of a user is written as the negative logarithm of the determinant of the MSE error covariance matrix, which is tightly related to the user MSE. Hence, minimizing the MSE of a user maximizes a tight lower bound on its rate. With (1), instead of considering each design criterion such as the MSE and the maximal mutual information in a separate way, a unifying framework can be developed. The link between most practical objective functions and the main diagonal elements of the MSE matrix has been established in [49] for point-to-point multicarrier MIMO communications, and this work has been extended to multicarrier MIMO relay communications in [51].

Our extensive literature survey reveals that MSE-based optimization problems have been considered for many communication systems but not for FD cognitive radio systems. This work tries to fill this gap and reveals useful insights into FD cognitive radio systems via MSE-based optimization.

\section{B. Notation}

The following notations are used in this paper. Matrices and vectors are denoted as bold capital and lowercase letters, respectively. $(\cdot)^{T}$ is the transpose; $(\cdot)^{H}$ is the conjugate transpose. $\mathbb{E}\{\cdot\}$ means the statistical expectation; $\mathbf{I}_{N}$ is the $N$ by $N$ identity matrix; $\mathbf{0}_{N \times M}$ is the $N$ by $M$ zero matrix; $\operatorname{tr}\{\cdot\}$ is the trace; $|\cdot|$ is the determinant; $\operatorname{diag}(\mathbf{A})$ is the diagonal matrix with the same diagonal elements as $\mathbf{A} . \mathcal{C N}\left(\mu, \sigma^{2}\right)$ denotes a complex Gaussian distribution with mean $\mu$ and variance $\sigma^{2}$. vec $(\cdot)$ stacks the elements of a matrix to one long column vector. The operator $\otimes$ denotes Kronecker product and $\perp$ denotes the statistical independence. $\|\cdot\|_{2}$ is the Euclidean norm of a vector. $\left\lfloor\mathbf{A}_{i}\right\rfloor_{i=1, \ldots, K}$ denotes a tall matrix (or vector) obtained by stacking the matrices $\mathbf{A}_{i}, i=1, \ldots, K$. 


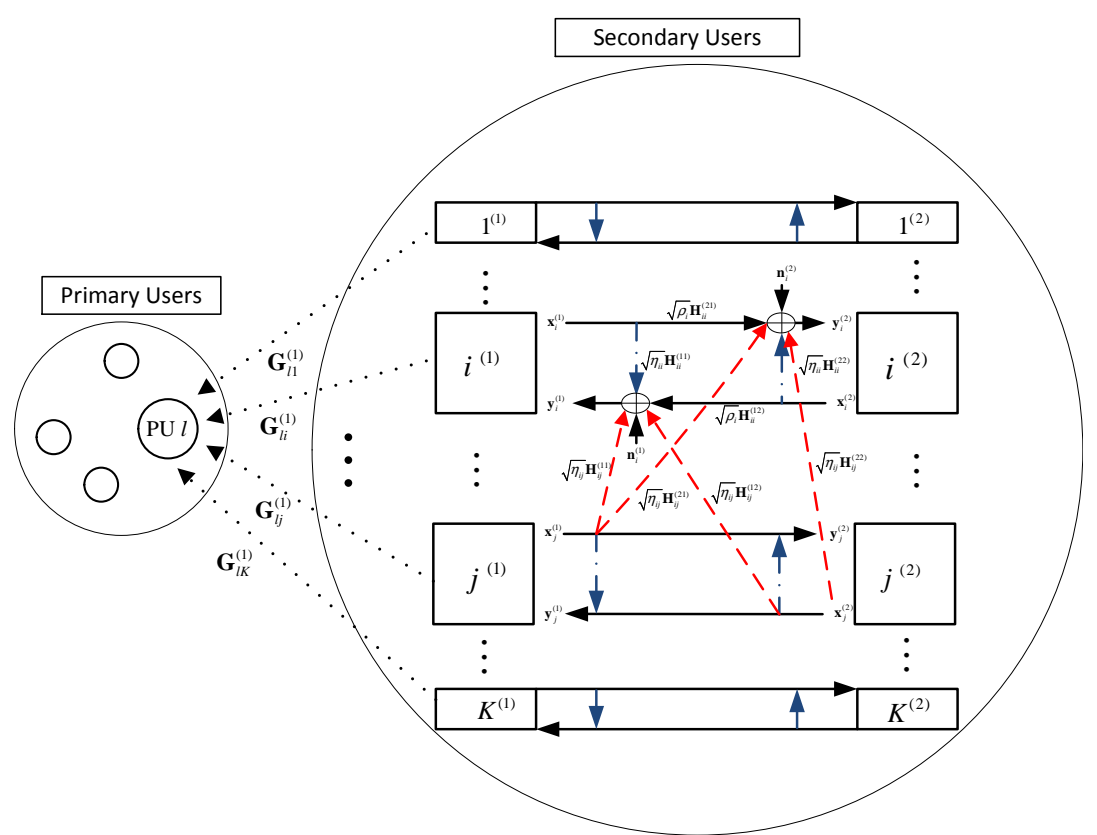

Fig. 1. Full-duplex MIMO cognitive radio system. Square and circle nodes denote the SUs and PUs, respectively. Dashed lines denote the interference between different pairs, dashed with dotted lines denote the self-interference, and dotted lines denote the interference from SUs to PUs.

\section{SYSTEM MODEL}

In this section, we describe the system model of a FD MIMO cognitive radio system, in which $K$ pair of SUs communicate simultaneously within the service range of $L$ PUs as seen in Fig. 1. Let us denote the set of SU pairs with $\mathcal{K} \triangleq\{1, \ldots, K\}$. The signals mentioned below are defined in complex baseband. We consider MIMO wireless channels, where all SUs are equipped with multiple antennas, and exchange information simultaneously with their pairs in a two way communication. We assume that the SU nodes in $i$ th link have $N_{i}$ and $M_{i}$ transmit and receive antennas, respectively.

We also take into account the limited dynamic range (DR), which is caused by non-ideal amplifiers, oscillators, ADCs, and digital-to-analog converters (DACs). We adopt the limited DR model in [17], which has also been commonly used in [21]-[25]. Particularly, at each receive antenna an additive white Gaussian "receiver distortion" with variance $\beta$ times the energy of the undistorted received signal on that receive antenna is applied, and at each transmit antenna, an additive white Gaussian "transmitter noise" with variance $\kappa$ times the energy of the intended transmit signal is applied. This transmitter/receiver distortion model is valid, since it was shown by hardware measurements in [52] and [53] that the non-ideality of the transmitter and receiver chain can be approximated by an independent Gaussian noise model, respectively.

The SU $i^{(a)}, i \in \mathcal{K}, a \in\{1,2\}$ receives signals from all the SU transmitters in the system via MIMO channels. $\mathbf{H}_{i i}^{(a b)} \in$ $\mathbb{C}^{M_{i} \times N_{i}}$ is the desired channel between node $a$ and $b$ of the $i$ th SU transmitter-receiver pair, where $b \in\{1,2\}$ and $b \neq a$. $\mathbf{H}_{i i}^{(a a)} \in \mathbb{C}^{M_{i} \times N_{i}}, a \in\{1,2\}$ denotes the self-interference channel of the SU $i^{(a)} \cdot \mathbf{H}_{i j}^{(a b)} \in \mathbb{C}^{M_{i} \times N_{j}},(a, b) \in\{1,2\}$ denotes the interference channel from the transmitter antennas of the SU $b$ in the $j$ th pair to the receiver antennas of the SU $a$ in the $i$ th pair, $(i, j) \in \mathcal{K}$ and $j \neq i$. All the channel matrices are assumed to be mutually independent, and the entries of each matrix are independent and identically distributed (i.i.d.) circular complex Gaussian variables with zero mean, independent real and imaginary parts, each with variance $1 / 2$. Particularly, each entry of all channel matrices has a uniform phase and Rayleigh magnitude, which models a Rayleigh fading environment [54].

The transmitted data streams of size $d_{i}$ at the $\mathrm{SU} i^{(a)}$ is denoted as $\mathbf{d}_{i}^{(a)} \in \mathbb{C}^{d_{i}}, i \in \mathcal{K}, a \in\{1,2\}$, and are assumed to be complex, zero mean, i.i.d. with

$$
\begin{aligned}
\mathbb{E}\left\{\mathbf{d}_{i}^{(a)}\right\} & =\mathbf{0}_{d_{i} \times 1}, \\
\mathbb{E}\left\{\mathbf{d}_{i}^{(a)}\left(\mathbf{d}_{j}^{(b)}\right)^{H}\right\} & = \begin{cases}\mathbf{I}_{d_{i}} & i=j \text { and } a=b \\
\mathbf{0}_{d_{i} \times d_{j}} & i \neq j \text { or } a \neq b .\end{cases}
\end{aligned}
$$

The $N_{i} \times 1$ signal vector transmitted by the $\mathrm{SU} i^{(a)}$ is given by

$$
\mathbf{x}_{i}^{(a)}=\mathbf{V}_{i}^{(a)} \mathbf{d}_{i}^{(a)}, \quad i \in \mathcal{K}, a \in\{1,2\},
$$

where $\mathbf{V}_{i}^{(a)} \in \mathbb{C}^{N_{i} \times d_{i}}$ represents the transmit beamforming matrix, and $\mathbf{x}_{i}^{(a)}$ is assumed to be Gaussian distributed with zero mean and covariance matrix as ${ }^{1}$

$$
\mathbb{E}\left\{\mathbf{x}_{i}^{(a)}\left(\mathbf{x}_{i}^{(a)}\right)^{H}\right\}=\mathbf{V}_{i}^{(a)}\left(\mathbf{V}_{i}^{(a)}\right)^{H} .
$$

We consider a FD MIMO interference channel between SUs that suffers from self-interference and interference from other pairs. Thus, the $\mathrm{SU} i^{(a)}$ receives a combination of the signals

${ }^{1}$ We assume that each SU uses a Gaussian codebook, since the Gaussian inputs are theoretically optimal, and are capacity achieving [54]. Since Gaussian inputs cannot be realized, discrete modulations/constellations are used in practice, but the performance of discrete constellations are far from that of ideal Gaussian inputs. Interested readers can refer to [55] in which the precoder designs under discrete constellations have been considered. 
transmitted by all the transmitters and noise. The $M_{i} \times 1$ received signal at the $\mathrm{SU} i^{(a)}$ is written as

$$
\begin{aligned}
\mathbf{y}_{i}^{(a)}= & \sqrt{\rho}_{i} \mathbf{H}_{i i}^{(a b)}\left(\mathbf{x}_{i}^{(b)}+\mathbf{c}_{i}^{(b)}\right)+\sqrt{\eta}_{i i} \mathbf{H}_{i i}^{(a a)}\left(\mathbf{x}_{i}^{(a)}+\mathbf{c}_{i}^{(a)}\right) \\
& +\sum_{j \neq i}^{K} \sum_{c=1}^{2} \sqrt{\eta_{i j}} \mathbf{H}_{i j}^{(a c)}\left(\mathbf{x}_{j}^{(c)}+\mathbf{c}_{j}^{(c)}\right)+\mathbf{e}_{i}^{(a)} \\
& +\mathbf{n}_{i}^{(a)}, i \in \mathcal{K}, \quad(a, b) \in\{1,2\} \text { and } a \neq b
\end{aligned}
$$

Here, $\mathbf{n}_{i}^{(a)} \in \mathbb{C}^{M_{i}}$ is the additive white Gaussian noise (AWGN) vector at $\mathrm{SU} i^{(a)}$ with zero mean and unit covariance matrix, and it is uncorrelated to all the transmitted signals ${ }^{2}$. In (5), $\rho_{i}$ denotes the average power gain of the $i$ th SU transmitter-receiver pair, $\eta_{i i}$ denotes the average power gain of the self-interference channel at the $i$ th SU pair, and $\eta_{i j}$ denotes the average power gain of the interference channel between the nodes at the $i$ th and $j$ th SU pair.

In (5), $\mathbf{c}_{i}^{(a)} \in \mathbb{C}^{N_{i}}, i \in \mathcal{K}, a \in\{1,2\}$ is the transmitter noise at the transmitter antennas of the $\mathrm{SU} i^{(a)}$, which models the effect of limited transmitter DR, and closely approximates the effects of additive power-amplifier noise, non-linearities in the DAC and phase noise. The covariance matrix of $\mathbf{c}_{i}^{(a)}$ is given by $\kappa(\kappa \ll 1)$ times the energy of the intended signal at each transmit antenna [17]. In particular $\mathbf{c}_{i}^{(a)}$ can be modeled as

$$
\begin{aligned}
& \mathbf{c}_{i}^{(a)} \sim \mathcal{C N}\left(\mathbf{0}, \kappa \operatorname{diag}\left(\mathbf{V}_{i}^{(a)}\left(\mathbf{V}_{i}^{(a)}\right)^{H}\right)\right), \\
& \mathbf{c}_{i}^{(a)} \perp \mathbf{x}_{i}^{(a)}
\end{aligned}
$$

In (5), $\mathbf{e}_{i}^{(a)} \in \mathbb{C}^{M_{i}}, i \in \mathcal{K}, a \in\{1,2\}$ is the additive receiver distortion at the receiver antennas of the $\mathrm{SU} i^{(a)}$, which models the effect of limited receiver DR, and closely approximates the combined effects of additive gain-control noise, non-linearities in the ADC and phase noise. The covariance matrix of $\mathbf{e}_{i}^{(a)}$ is given by $\beta(\beta \ll 1)$ times the energy of the undistorted received signal at each receive antenna [17]. In particular, $\mathbf{e}_{i}^{(a)}$ can be modeled as

$$
\begin{aligned}
& \mathbf{e}_{i}^{(a)} \sim \mathcal{C N}\left(\mathbf{0}, \beta \operatorname{diag}\left(\boldsymbol{\Phi}_{i}^{(a)}\right)\right), \\
& \mathbf{e}_{i}^{(a)} \perp \mathbf{u}_{i}^{(a)},
\end{aligned}
$$

where $\boldsymbol{\Phi}_{i}^{(a)}=\operatorname{Cov}\left\{\mathbf{u}_{i}^{(a)}\right\}$ and $\mathbf{u}_{i}^{(a)}$ is the undistorted received vector at the SU $i^{(a)}$, i.e., $\mathbf{u}_{i}^{(a)}=\mathbf{y}_{i}^{(a)}-\mathbf{e}_{i}^{(a)}$.

The SU $i^{(a)}$ knows the interfering codewords $\mathbf{x}_{i}^{(a)}$, and its self-interference channel $\mathbf{H}_{i i}^{(a a)}$, so the self-interference term $\sqrt{\eta}{ }_{i i} \mathbf{H}_{i i}^{(a a)} \mathbf{x}_{i}^{(a)}$ is known, and thus can be canceled [17] $]^{3}$. The

\footnotetext{
${ }^{2}$ Since the SU receiver cannot differentiate the interference generated by the PUs from the background thermal noise, the noise vector $\mathbf{n}_{i}^{(a)}$ in (5) captures the background thermal noise as well as the interference generated by the PUs. This assumption is also adopted in [32]-[37].

${ }^{3}$ The channel state information (CSI) of the self-interference channel can be acquired by using pilot signals. Since the pilot signal of a FD node is echoed back to itself, and the received power of this echoed-backed pilot signal is very high (due to small distances between transmit and receive antennas of a node), the self-interference channel can be estimated with high accuracy [1].
}

interference canceled signal can then be written as ${ }^{4}$

$$
\begin{aligned}
\tilde{\mathbf{y}}_{i}^{(a)} & =\mathbf{y}_{i}^{(a)}-\sqrt{\eta}_{i i} \mathbf{H}_{i i}^{(a a)} \mathbf{x}_{i}^{(a)} \\
& =\sqrt{\rho}_{i} \mathbf{H}_{i i}^{(a b)} \mathbf{x}_{i}^{(b)}+\mathbf{v}_{i}^{(a)},
\end{aligned}
$$

where $\mathbf{v}_{i}^{(a)}$ is the residual interference components of (10) after self-interference cancellation and is given by

$$
\begin{aligned}
\mathbf{v}_{i}^{(a)}= & \sqrt{\rho}_{i} \mathbf{H}_{i i}^{(a b)} \mathbf{c}_{i}^{(b)}+\sqrt{\eta}_{i i} \mathbf{H}_{i i}^{(a a)} \mathbf{c}_{i}^{(a)}+\mathbf{e}_{i}^{(a)}+\mathbf{n}_{i}^{(a)} \\
& +\sum_{j \neq i}^{K} \sum_{c=1}^{2} \sqrt{\eta_{i j}} \mathbf{H}_{i j}^{(a c)}\left(\mathbf{x}_{j}^{(c)}+\mathbf{c}_{j}^{(c)}\right)
\end{aligned}
$$

Using (6)-(9), similar to [17], $\boldsymbol{\Sigma}_{i}^{(a)}$, the covariance matrix of $\mathbf{v}_{i}^{(a)}$, can be approximated as in (12) shown at the bottom of the following page ${ }^{5}$.

We assume that the $\mathrm{SU} i^{(a)}$ applies the linear receiver $\mathbf{R}_{i}^{(a)} \in \mathbb{C}^{d_{i} \times M_{i}}$ to estimate the signal transmitted from SU $i^{(b)}$. That is

$$
\begin{aligned}
\hat{\mathbf{d}}_{i}^{(b)} & =\mathbf{R}_{i}^{(a)} \tilde{\mathbf{y}}_{i}^{(a)} \\
& =\sqrt{\rho} \mathbf{R}_{i}^{(a)} \mathbf{H}_{i i}^{(a b)} \mathbf{V}_{i}^{(b)} \mathbf{d}_{i}^{(b)}+\mathbf{R}_{i}^{(a)} \mathbf{v}_{i}^{(a)}
\end{aligned}
$$

We can now formulate the MSE of the SU $i^{(a)}$. Using (13), the MSE matrix of the SU $i^{(a)}$ can be written as

$$
\begin{aligned}
\mathbf{M S E}_{i}^{(a)} & \\
= & \mathbb{E}\left\{\left(\hat{\mathbf{d}}_{i}^{(b)}-\mathbf{d}_{i}^{(b)}\right)\left(\hat{\mathbf{d}}_{i}^{(b)}-\mathbf{d}_{i}^{(b)}\right)^{H}\right\} \\
= & \left(\sqrt{\rho}_{i} \mathbf{R}_{i}^{(a)} \mathbf{H}_{i i}^{(a b)} \mathbf{V}_{i}^{(b)}-\mathbf{I}_{d_{i}}\right)\left(\sqrt{\rho}_{i} \mathbf{R}_{i}^{(a)} \mathbf{H}_{i i}^{(a b)} \mathbf{V}_{i}^{(b)}-\mathbf{I}_{d_{i}}\right)^{H} \\
& +\mathbf{R}_{i}^{(a)} \boldsymbol{\Sigma}_{i}^{(a)}\left(\mathbf{R}_{i}^{(a)}\right)^{H} .
\end{aligned}
$$

As mentioned before, the SUs are installed within the service range of $L$ PUs, for which the SUs should provide protection. We assume that each $\mathrm{PU}$ is equipped with $N$ receive antennas. The received interference signal at the $l$ th PU from SUs is expressed as

$\mathbf{y}_{l}^{P U}=\sum_{i=1}^{K} \sum_{b=1}^{2} \sqrt{\mu_{l i}^{(b)}} \mathbf{G}_{l i}^{(b)}\left(\mathbf{x}_{i}^{(b)}+\mathbf{c}_{i}^{(b)}\right), l=1, \ldots, L$,

where $\mathbf{G}_{l i}^{(b)} \in \mathbb{C}^{N \times N_{i}}$ is the channel between the lth PU and $i^{(b)}$ th $\mathbf{S U}$, which is modeled similar to $\mathbf{H}_{i j}^{(a b)}$ discussed in Section II, and $\mu_{l i}^{(b)}$ is the average power gain of $\mathbf{G}_{l i}^{(b)}$. Using (15), the power of the interference resulting from SUs

\footnotetext{
${ }^{4}$ In practice, even if the self-interference is suppressed to some extent using analog and digital self-interference cancellation techniques, due to transmitter $\left(\mathbf{c}_{i}^{(a)}\right)$ and receiver $\left(\mathbf{e}_{i}^{(a)}\right)$ distortion, the self-interference cannot be canceled completely resulting in residual self-interference. Depending on the strength of the residual self-interference, optimal transmit strategies for HD systems, can depart from optimal. If the residual self-interference is not well managed, it can still prevent us from exploiting the benefits of FD systems.

${ }^{5}$ Note that $(12)$ is approximated under $\kappa \ll 1$ and $\beta \ll 1$, which is a practical assumption [16], [17]. Therefore, the terms including the multiplication of $\kappa$ and $\beta$ are negligible, and have been ignored in the approximation.
} 
at the $l$ th PU can be written as

$$
\begin{aligned}
I_{l}^{P U}=\sum_{i=1}^{K} & \sum_{b=1}^{2} \mu_{l i}^{(b)} \operatorname{tr}\left\{\mathbf { G } _ { l i } ^ { ( b ) } \left(\mathbf{V}_{i}^{(b)}\left(\mathbf{V}_{i}^{(b)}\right)^{H}\right.\right. \\
& \left.\left.+\kappa \operatorname{diag}\left(\mathbf{V}_{i}^{(b)}\left(\mathbf{V}_{i}^{(b)}\right)^{H}\right)\right)\left(\mathbf{G}_{l i}^{(b)}\right)^{H}\right\} .
\end{aligned}
$$

Note that underlay cognitive radio systems enable SUs to transmit with overlapping spectrum with PUs as long as the QoS of PUs is not degraded. This is managed by, e.g., introducing some interference constraints that impose upper bounds on the total aggregate interference induced by all SUs to each PU. The choice of this upper bound (or threshold) is a complex and open regulatory issue, which can be the result of a negotiation or opportunistic-based procedure between PUs (or regulatory agencies) and SUs [32]. Both deterministic and probabilistic interference constraints have been suggested in the literature [30], [31]. In this paper, we will consider deterministic interference constraints as assumed in [32]-[34]. Particularly, we assume that the PU imposing the interference constraint, has already computed its maximum tolerable interference threshold.

Channel estimation between SUs can be accomplished using standard signal processing techniques via training through the pilots and feedback [38]. Channel estimation between SUs and PUs is more challenging, because PUs are unlikely to cooperate with SUs. But, if the primary system adopts the TDD scheme, by exploiting the channel reciprocity, channel between SUs and PUs can be acquired at the SUs by overhearing the transmissions between PU transmitter and receiver pair [38]-[42]. If TDD scheme is not feasible, blind beamforming techniques can be employed [38]. Other methods to acquire the CSI knowledge between PUs and SUs is 1) through environmental learning [43], [44], 2) by exchange of CSI between the PUs and SUs through a band manager, which mediates between the two parties [39], [40], [45], and 3) the primary system can cooperate with the secondary system to exchange the channel estimates [38].

\section{SUM-MSE MinimizATION}

We take Sum-MSE as the performance measure to design the transceivers. Upper limits on both transmit power of the SUs and interfering power at the PUs are considered. Using the following definition for a stacked matrix $\overline{\mathbf{V}}: \overline{\mathbf{V}}=$
$\left[\overline{\mathbf{V}}_{1}^{T}, \ldots, \overline{\mathbf{V}}_{K}^{T}\right]^{T}$ with $\overline{\mathbf{V}}_{k}=\left[\left(\mathbf{V}_{k}^{(1)}\right)^{T},\left(\mathbf{V}_{k}^{(2)}\right)^{T}\right]^{T}$, Sum-

MSE optimization scheme is formulated as follows

$$
\begin{array}{ll}
\min _{\overline{\mathbf{V}}, \overline{\mathbf{R}}} & \sum_{i=1}^{K} \sum_{a=1}^{2} \operatorname{tr}\left\{\mathbf{M S E}_{i}^{(a)}\right\} \\
\text { s.t. } & \operatorname{tr}\left\{\mathbf{V}_{i}^{(b)}\left(\mathbf{V}_{i}^{(b)}\right)^{H}\right\} \leq P_{i}^{(b)}, i \in \mathcal{K}, b=1,2 \\
& I_{l}^{P U} \leq \lambda_{l}, l=1, \ldots, L,
\end{array}
$$

where $P_{i}^{(b)}$ is the power constraint at the $i^{(b)}$ th SU transmitter, and $\lambda_{l}$ is the upper bound of the interference allowed to be imposed on the $l$ th PU.

Note that the Sum-MSE function (17) is not jointly convex over transmit beamforming matrices $\overline{\mathbf{V}}$ and receiving beamforming matrices $\overline{\mathbf{R}}$ (since they are coupled), but is component-wise convex over $\overline{\mathbf{V}}$ and $\overline{\mathbf{R}}$. Since it is not jointly convex, we cannot apply the standard convex optimization methods to obtain the optimal solution. Therefore, we will employ an iterative algorithm that finds the efficient solutions of $\overline{\mathbf{V}}$ and $\overline{\mathbf{R}}$ in an alternating fashion. Particularly, we update the transmit beamforming matrices $\overline{\mathbf{V}}$ when the receiving beamforming matrices $\overline{\mathbf{R}}$ are fixed, and then using $\overline{\mathbf{V}}$ obtained at the previous step, we update the receiving beamforming matrices $\overline{\mathbf{R}}$. The iterations continue until convergence or a pre-defined number of iterations is reached.

Under the fixed transmit beamforming matrices, the optimal receive beamforming matrices at the $\mathrm{SU} i^{(a)}$ is MMSE receiver filter which can be expressed as

$$
\begin{aligned}
& \mathbf{R}_{i}^{(a) *}=\underset{\mathbf{R}_{i}^{(a)}}{\arg \min } \operatorname{tr}\left\{\mathbf{M S E}_{i}^{(a)}\right\} \\
& =\sqrt{\rho_{i}}\left(\mathbf{V}_{i}^{(b)}\right)^{H}\left(\mathbf{H}_{i i}^{(a b)}\right)^{H} \\
& \times\left(\rho_{i} \mathbf{H}_{i i}^{(a b)} \mathbf{V}_{i}^{(b)}\left(\mathbf{V}_{i}^{(b)}\right)^{H}\left(\mathbf{H}_{i i}^{(a b)}\right)^{H}+\boldsymbol{\Sigma}_{i}^{(a)}\right)^{-1} \text {. }
\end{aligned}
$$

Under the fixed receive beamforming matrices, the optimum transmit beamforming matrices are found as follows. Using epigraph form and introducing slack variables $\tau_{i}^{(a)}$, (17)-(19)

$$
\begin{aligned}
\boldsymbol{\Sigma}_{i}^{(a)} \approx & \rho_{i} \kappa \mathbf{H}_{i i}^{(a b)} \operatorname{diag}\left(\mathbf{V}_{i}^{(b)}\left(\mathbf{V}_{i}^{(b)}\right)^{H}\right)\left(\mathbf{H}_{i i}^{(a b)}\right)^{H}+\eta_{i i} \kappa \mathbf{H}_{i i}^{(a a)} \operatorname{diag}\left(\mathbf{V}_{i}^{(a)}\left(\mathbf{V}_{i}^{(a)}\right)^{H}\right)\left(\mathbf{H}_{i i}^{(a a)}\right)^{H} \\
& +\beta \rho_{i} \operatorname{diag}\left(\mathbf{H}_{i i}^{(a b)} \mathbf{V}_{i}^{(b)}\left(\mathbf{V}_{i}^{(b)}\right)^{H}\left(\mathbf{H}_{i i}^{(a b)}\right)^{H}\right)+\beta \eta_{i i} \operatorname{diag}\left(\mathbf{H}_{i i}^{(a a)} \mathbf{V}_{i}^{(a)}\left(\mathbf{V}_{i}^{(a)}\right)^{H}\left(\mathbf{H}_{i i}^{(a a)}\right)^{H}\right) \\
& +\sum_{j \neq i}^{K} \sum_{c=1}^{2} \eta_{i j}\left[\mathbf{H}_{i j}^{(a c)}\left(\mathbf{V}_{j}^{(c)}\left(\mathbf{V}_{j}^{(c)}\right)^{H}+\kappa \operatorname{diag}\left(\mathbf{V}_{j}^{(c)}\left(\mathbf{V}_{j}^{(c)}\right)^{H}\right)\left(\mathbf{H}_{i j}^{(a c)}\right)^{H}\right]\right. \\
& +\sum_{j \neq i}^{K} \sum_{c=1}^{2} \beta \eta_{i j} \operatorname{diag}\left(\mathbf{H}_{i j}^{(a c)} \mathbf{V}_{j}^{(c)}\left(\mathbf{V}_{j}^{(c)}\right)^{H}\left(\mathbf{H}_{i j}^{(a c)}\right)^{H}\right)+\mathbf{I}_{M_{i} .}
\end{aligned}
$$


is rewritten as

$$
\begin{array}{ll}
\min _{\overline{\mathbf{V}}, \tau_{i}^{(a)}} & \sum_{i=1}^{K} \sum_{a=1}^{2} \tau_{i}^{(a)} \\
\text { s.t. } & \operatorname{tr}\left\{\mathbf{V}_{i}^{(b)}\left(\mathbf{V}_{i}^{(b)}\right)^{H}\right\} \leq P_{i}^{(b)}, i \in \mathcal{K}, b=1,2,(22) \\
& I_{l}^{P U} \leq \lambda_{l}, l=1, \ldots, L \\
& \operatorname{tr}\left\{\mathbf{M S E}_{i}^{(a)}\right\} \leq \tau_{i}^{(a)}, i \in \mathcal{K}, a=1,2
\end{array}
$$

To solve the optimization problem (21)-(24), we need to write $\operatorname{tr}\left\{\mathbf{M S E}_{i}^{(a)}\right\}$ and $I_{l}^{P U}$ in vector form. As shown in Appendix, the vector forms of $\operatorname{tr}\left\{\mathbf{M S E}_{i}^{(a)}\right\}$ and $I_{l}^{P U}$ can be written as in (25) and (26), respectively given at the bottom of the following page.

Using the vector forms, the optimization problem (21)-(24) can be rewritten as

$$
\begin{array}{cl}
\min _{\overline{\mathbf{V}}, \tau_{i}^{(a)}} & \sum_{i=1}^{K} \sum_{a=1}^{2} \tau_{i}^{(a)} \\
\text { s.t. } & \left\|\operatorname{vec}\left(\mathbf{V}_{i}^{(b)}\right)\right\|_{2}^{2} \leq P_{i}^{(b)}, i \in \mathcal{K}, b=1,2, \\
& \left\|\boldsymbol{\alpha}_{l}\right\|_{2}^{2} \leq \lambda_{l}, l=1, \ldots, L \\
& \left\|\boldsymbol{\mu}_{i}^{(a)}\right\|_{2}^{2} \leq \tau_{i}^{(a)}, i \in \mathcal{K}, a=1,2 .
\end{array}
$$

Since the objective function (27) is linear, and the constraints (28)-(30) are second-order cones, (27)-(30) is a second-order cone programming (SOCP) problem [56], and can be efficiently solved by standard SOCP solvers with polynomial complexity using interior point methods [57].

\section{A. Discussion}

The algorithm for the Sum-MSE optimization problem (17)(19) is given in Table I. Since the proposed Sum-MSE algorithm monotonically decreases the total MSE over each iteration by updating the transceivers in an alternating fashion, and the fact that MSE is bounded below (at least by zero), it is clear that the proposed Sum-MSE algorithm is convergent and is guaranteed to converge to a local minimum. Since the Sum-MSE optimization problem is not jointly convex, the proposed algorithm is not guaranteed to converge to a global optimum point. Therefore, good initialization points should be selected to ensure a suboptimal solution with a good performance. In the simulations, we use right singular matrices as initialization [46].

Assuming the same number of transmit antennas, receive antennas, and data streams at each node, i.e. $N_{i}=N$, $M_{i}=N$, and $d_{i}=N$, the complexity of the proposed algorithm is computed as follows. The update of $\mathbf{R}_{i}^{(a)}$ requires $\mathcal{O}\left(8 N^{3} K\right)$ for the matrix multiplications inside the inverse, $\mathcal{O}\left(N^{3}\right)$ for the inverse, and $\mathcal{O}\left(2 N^{3}\right)$ for the matrix multiplications outside of the inverse. The complexity of computing the optimal transmit beamforming mainly depends on solving SOCP problem. The number of iterations required to solve a SOCP problem using interior point methods can be computed according to [56]. For a SOCP with $n$ variables and $m$ conic constraints with dimension $m_{i}$ each,
TABLE I

MSE-BASEd TRANSCEIVER DESIGNS

1) Set the iteration number $n=0$ and initialize $\overline{\mathbf{V}}^{[n]}$.

2) $n \leftarrow n+1$. Update $\left(\mathbf{R}_{i}^{(a)}\right)^{[n]}$ using (20).

3) Update $\left(\mathbf{V}_{i}^{(b)}\right)^{[n]}$ by solving (27)-(30)

4) Repeat steps 2 and 3 until convergence or a predefined number of iterations is reached.

its complexity scales by $\mathcal{O}\left(n^{2} \sum_{m} m_{i}\right)$. Therefore, the update of $\mathbf{V}_{i}^{(b)}$ requires a total computational complexity of $\mathcal{O}\left(8 K^{3}\left(N^{2}+1\right)^{2}\left(N^{2}+2 L N^{2}+N^{2}(6 K-1)+1\right)\right)$.

The proposed algorithm requires a central scheduler, which coordinates the calibration of channel matrices, collects all channel matrices, and then computes and distributes the beamforming matrices of all links. In a cellular system, the BS can take up the role of the scheduler. In an interference channel (or an ad-hoc network), the scheduler can reside at any node in the network. In a dynamic environment, the scheduler can be adaptively elected among the eligible nodes in the network [58]-[60]. The election can be done based on the capacity of a node, the status of a node, and the location of a node, etc. The research of the scheduler election issues is important but beyond the scope of this paper. We assume that a scheduler is available for the network within the time scale of interest.

\section{EXTENSIONS}

\section{A. Min-Max MSE Minimization}

Unlike the minimum Sum-MSE transceiver design discussed in Section III, the Min-Max MSE transceiver design ensures each receiver has the same MSE so that it introduces fairness among the nodes, i.e., it guarantees a certain level of fairness among the nodes. The Min-Max MSE optimization problem can be formulated as:

$$
\begin{array}{ll}
\min _{\overline{\mathbf{V}}, \overline{\mathbf{R}}} \max _{i \in \mathcal{K}, a=1,2} & \operatorname{tr}\left\{\mathbf{M S E}_{i}^{(a)}\right\} \\
\text { s.t. } & \operatorname{tr}\left\{\mathbf{V}_{i}^{(b)}\left(\mathbf{V}_{i}^{(b)}\right)^{H}\right\} \leq P_{i}^{(b)}, \forall(i, b),(32 \\
& I_{l}^{P U} \leq \lambda_{l}, l=1, \ldots, L
\end{array}
$$

Similar to the Sum-MSE optimization problem (17)-(19), the Min-Max MSE optimization problem is not jointly convex over transmit beamforming matrices $\overline{\mathbf{V}}$ and receiving beamforming matrices $\overline{\mathbf{R}}$. Therefore we carry out the optimization procedure iteratively in an alternating fashion.

Under the fixed values of the transmit beamforming matrices $\overline{\mathbf{V}}$, the optimal receiving beamforming matrix, $\mathbf{R}_{i}^{(a)}, i \in$ $\mathcal{K}, a=1,2$ is linear MMSE receiver given in (20). Under the fixed receiving beamforming matrices $\mathbf{R}_{i}^{(a)}$, with the introduction of an auxiliary variable $t$, which is an upper bound on the square root of $\operatorname{tr}\left\{\mathbf{M S E}_{i}^{(a)}\right\}$ (i.e., $\sqrt{\operatorname{tr}\left\{\mathbf{M S E} \mathbf{S}_{i}^{(a)}\right\}} \leq t, \forall i \in$ $\mathcal{K}, a=1,2)$, the Min-Max optimization problem (31)-(33) 
can be written as

$$
\begin{array}{ll}
\min _{\overline{\mathbf{V}}, t} & t \\
\text { s.t. } & \sqrt{\operatorname{tr}\left\{\mathbf{M S E}_{i}^{(a)}\right\}} \leq t, i \in \mathcal{K}, a=1,2 \\
& \operatorname{tr}\left\{\mathbf{V}_{i}^{(b)}\left(\mathbf{V}_{i}^{(b)}\right)^{H}\right\} \leq P_{i}^{(b)}, i \in \mathcal{K}, b=1,2, \\
& I_{l}^{P U} \leq \lambda_{l}, l=1, \ldots, L .
\end{array}
$$

With the vector forms in (25) and (26), the Min-Max optimization problem of transmit beamforming matrices (34)(37) can be written as

$$
\begin{array}{cl}
\min _{\overline{\mathbf{V}}, t} & t \\
\text { s.t. } & \left\|\boldsymbol{\mu}_{i}^{(a)}\right\|_{2} \leq t, i \in \mathcal{K}, a=1,2, \\
& \left\|\operatorname{vec}\left(\mathbf{V}_{i}^{(b)}\right)\right\|_{2} \leq \sqrt{P_{i}^{(b)}}, i \in \mathcal{K}, b=1,2, \\
& \left\|\boldsymbol{\alpha}_{l}\right\|_{2}^{2} \leq \lambda_{l}, l=1, \ldots, L .
\end{array}
$$

Similar to (27)-(30), the optimization problem (38)-(41) is also a SOCP problem [56], and can be efficiently solved by standard SOCP solvers with polynomial complexity using interior point methods [57].

\section{B. Sum-power constrained transceiver design}

In this subsection, we consider sum-power constrained optimization problem, which will be applied in FD cellular systems discussed in Section V. Note that sum-power constraint is still important and motivated for interference channels under the emerging scenarios such as energy-harvesting-based communication systems, distributed antenna systems, games played by resource-constrained players, and fair comparison in heterogeneous networks [61].

Similar to the individual power constrained optimization problem, the proposed SOCP algorithm can also be applied for the sum-power constrained optimization problem given as:

$$
\begin{array}{ll}
\min _{\overline{\mathbf{V}}, \overline{\mathbf{R}}} & \sum_{i=1}^{K} \sum_{a=1}^{2} \operatorname{tr}\left\{\mathbf{M S E}_{i}^{(a)}\right\} \\
\text { s.t. } & \sum_{i=1}^{K} \sum_{b=1}^{2} \operatorname{tr}\left\{\mathbf{V}_{i}^{(b)}\left(\mathbf{V}_{i}^{(b)}\right)^{H}\right\} \leq P_{T}, \\
& I_{l}^{P U} \leq \lambda_{l}, l=1, \ldots, L,
\end{array}
$$

where $P_{T}$ is the total power constraint of the system. Using the vector forms in (25) and (26), the optimization problem (42)-

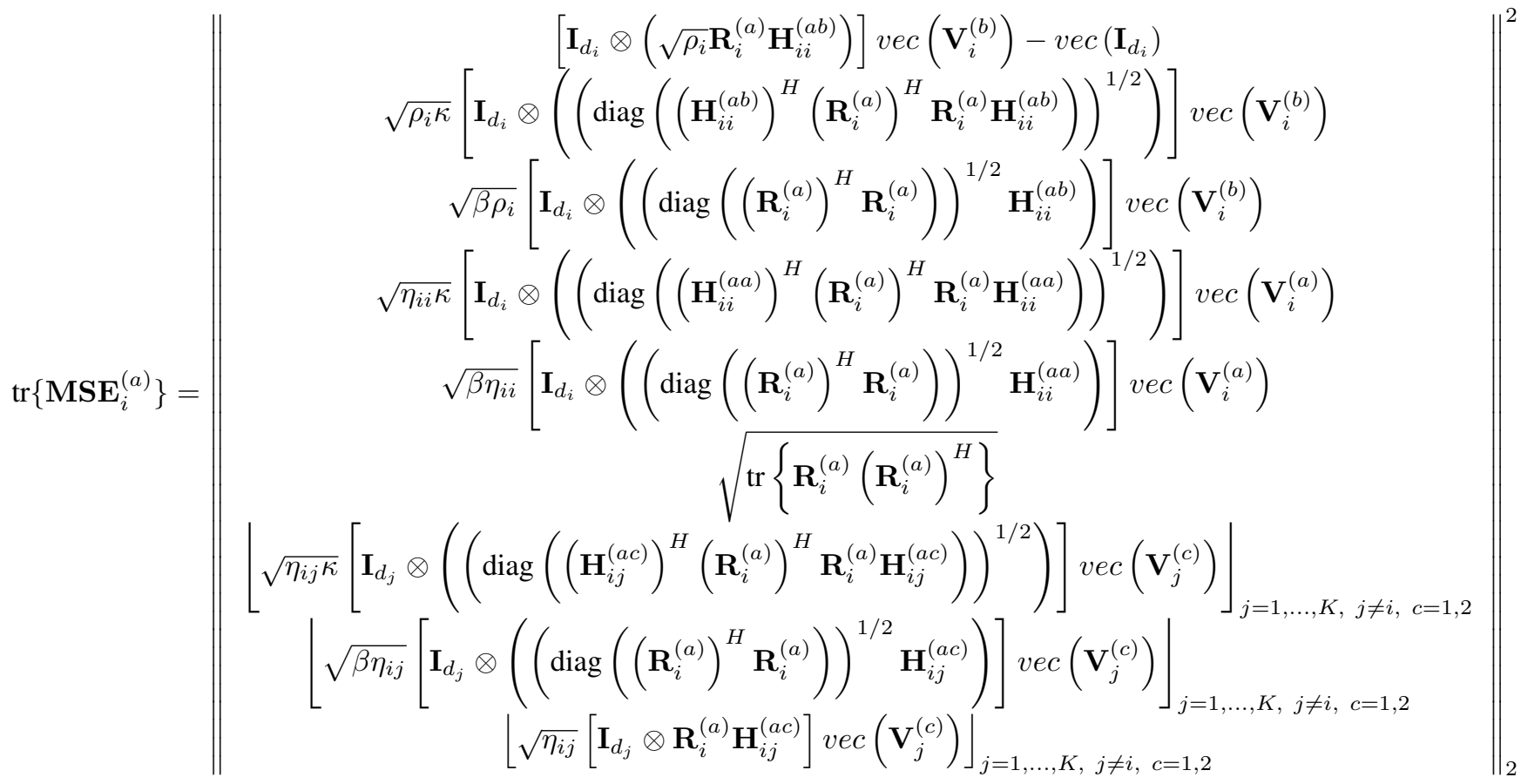

$$
\begin{aligned}
& \triangleq\left\|\boldsymbol{\mu}_{i}^{(a)}\right\|_{2}^{2} \text {. }
\end{aligned}
$$

$$
\begin{aligned}
I_{l}^{P U} & \left.=\|\left\lfloor\left.\sqrt{\mu_{l i}^{(b)} \kappa}\left[\mathbf{I}_{d_{i}} \otimes\left(\left(\operatorname{diag}\left(\left(\mathbf{G}_{l i}^{(b)}\right)^{H} \mathbf{G}_{l i}^{(b)}\right)\right)^{1 / 2}\right)\right] \operatorname{vec}\left(\mathbf{I}_{i}^{(b)}\right)\right|_{i=1, \ldots, K, b=1,2} \|_{2}^{(b)}\right] \operatorname{vec}\left(\mathbf{V}_{i}^{(b)}\right)\right]_{i=1, \ldots, K, b=1,2} \\
& \triangleq\left\|\boldsymbol{\alpha}_{l}\right\|_{2}^{2} .
\end{aligned}
$$


(44) can be rewritten, under the fixed receive beamforming matrices, as

$$
\begin{array}{ll}
\min _{\overline{\mathbf{V}}, \tau_{i}^{(a)}} & \sum_{i=1}^{K} \sum_{a=1}^{2} \tau_{i}^{(a)} \\
\text { s.t. } & \|\operatorname{vec}(\overline{\mathbf{V}})\|_{2}^{2} \leq P_{T}, \\
& \left\|\boldsymbol{\alpha}_{l}\right\|_{2}^{2} \leq \lambda_{l}, l=1, \ldots, L, \\
& \left\|\boldsymbol{\mu}_{i}^{(a)}\right\|_{2}^{2} \leq \tau_{i}^{(a)}, i \in \mathcal{K}, a=1,2 .
\end{array}
$$

Since the problem (45)-(48) is a SOCP problem, it can be efficiently solved by standard SOCP solvers.

\section{Full-Duplex Cognitive Cellular Systems}

In this section, we show that the algorithm proposed for the FD cognitive MIMO interference channel also holds for FD cognitive cellular systems, in which a FD BS communicates with HD mode UL and DL users, simultaneously as seen in Fig. 2. The BS serves $K$ UL users and $J$ DL users simultaneously. The BS is equipped with $M_{0}$ and $N_{0}$ transmit and receive antennas, respectively. The number of antennas of the $k$-th UL user and the $j$-th DL user are denoted by $M_{k}$ and $N_{j}$, respectively. The number of data streams transmitted from the $k$-th UL user (to the $j$-th DL user) is denoted by $d_{k}^{U L}\left(d_{j}^{D L}\right)$.

$\mathbf{H}_{k}^{U L} \in \mathbb{C}^{N_{0} \times M_{k}}$ and $\mathbf{H}_{j}^{D L} \in \mathbb{C}^{N_{j} \times M_{0}}$ represent the $k$-th UL channel and the $j$-th DL channel, respectively. $\mathbf{H}_{0} \in \mathbb{C}^{N_{0} \times M_{0}}$ is the self-interference channel from the transmitter antennas of BS to the receiver antennas of BS. $\mathbf{H}_{j k}^{D U} \in \mathbb{C}^{N_{j} \times M_{k}}$ denotes the CCI channel from the $k$-th UL user to the $j$-th DL user.

The vector of source symbols transmitted by the $k$-th UL user is denoted as $\mathbf{s}_{k}^{U L}=\left[s_{k, 1}^{U L}, \ldots, s_{k, d_{k}^{U L}}^{U L}\right]^{T}$. It is assumed that the symbols are i.i.d. with unit power, i.e., $\mathbb{E}\left[\mathbf{s}_{k}^{U L}\left(\mathbf{s}_{k}^{U L}\right)^{H}\right]=\mathbf{I}_{d_{k}^{U L}}$. Similarly, the transmit symbols for the $j$-th DL user is denoted by $\mathbf{s}_{j}^{D L}=\left[s_{j, 1}^{D L}, \ldots, s_{j, d_{j}^{D L}}^{D L}\right]^{T}$, with $\mathbb{E}\left[\mathbf{s}_{j}^{D L}\left(\mathbf{s}_{j}^{D L}\right)^{H}\right]=\mathbf{I}_{d_{j}^{D L}}$. Denoting the precoders for the data streams of the $k$-th UL and $j$-th DL user as $\mathbf{V}_{k}^{U L}=\left[\mathbf{v}_{k, 1}^{U L}, \ldots, \mathbf{v}_{k, d_{k}^{U L}}^{U L}\right] \in \mathbb{C}^{M_{k} \times d_{k}^{U L}}$, and $\mathbf{V}_{j}^{D L}=$ $\left[\mathbf{v}_{j, 1}^{D L}, \ldots, \mathbf{v}_{j, d_{j}^{D L}}^{D L}\right] \in \mathbb{C}^{M_{0} \times d_{j}^{D L}}$, respectively, the transmitted signal of the $k$-th UL user and that of the BS can be written, respectively, as

$$
\mathbf{x}_{k}^{U L}=\mathbf{V}_{k}^{U L} \mathbf{s}_{k}^{U L}, \quad \mathbf{x}_{0}=\sum_{j=1}^{J} \mathbf{V}_{j}^{D L} \mathbf{s}_{j}^{D L} .
$$

We consider a FD multi-user MIMO system that suffers from self-interference and CCI. The signal received by the BS and that received by the $j$-th DL user can be written, respectively, as

$$
\begin{aligned}
\mathbf{y}_{0}= & \sum_{k=1}^{K} \mathbf{H}_{k}^{U L}\left(\mathbf{x}_{k}^{U L}+\mathbf{c}_{k}^{U L}\right)+\mathbf{H}_{0}\left(\mathbf{x}_{0}+\mathbf{c}_{0}\right) \\
& +\mathbf{e}_{0}+\mathbf{n}_{0}, \\
\mathbf{y}_{j}^{D L}= & \mathbf{H}_{j}^{D L}\left(\mathbf{x}_{0}+\mathbf{c}_{0}\right)+\sum_{k=1}^{K} \mathbf{H}_{j k}^{D U}\left(\mathbf{x}_{k}^{U L}+\mathbf{c}_{k}^{U L}\right) \\
& +\mathbf{e}_{j}^{D L}+\mathbf{n}_{j}^{D L},
\end{aligned}
$$

where $\mathbf{n}_{0} \in \mathbb{C}^{N_{0}}$ and $\mathbf{n}_{j}^{D L} \in \mathbb{C}^{N_{j}}$ denote the AWGN vector with zero mean and unit covariance matrix at the the BS and the $j$-th DL user, respectively. In (50)-(51), $\mathbf{c}_{k}^{U L}\left(\mathbf{c}_{0}\right)$ is the transmitter distortion at the $k$-th UL user (BS), which is modeled as in (6)-(7), and $\mathbf{e}_{j}^{D L}\left(\mathbf{e}_{0}\right)$ is the receiver distortion at the $j$-th DL user (BS), which is modeled as in (8)-(9).

From (50)-(51), the aggregate interference-plus-noise terms at the $k$-th UL and the $j$-th DL user are written, respectively as

$$
\begin{aligned}
\mathbf{m}_{k}^{U L}= & \sum_{j=1, j \neq k}^{K} \mathbf{H}_{j}^{U L} \mathbf{x}_{j}^{U L}+\sum_{j=1}^{K} \mathbf{H}_{j}^{U L} \mathbf{c}_{j}^{U L}+\mathbf{H}_{0}\left(\mathbf{x}_{0}+\mathbf{c}_{0}\right) \\
& +\mathbf{e}_{0}+\mathbf{n}_{0}, k=1, \ldots, K, \\
\mathbf{m}_{j}^{D L}= & \mathbf{H}_{j}^{D L} \sum_{k=1, k \neq j}^{J} \mathbf{V}_{k}^{D L} \mathbf{s}_{k}^{D L}+\sum_{k=1}^{K} \mathbf{H}_{j k}^{D U}\left(\mathbf{x}_{k}^{U L}+\mathbf{c}_{k}^{U L}\right) \\
& +\mathbf{H}_{j}^{D L} \mathbf{c}_{0}+\mathbf{e}_{j}^{D L}+\mathbf{n}_{j}^{D L}, j=1, \ldots, J .
\end{aligned}
$$

Similar to [17], the covariance matrix of $\mathbf{m}_{k}^{U L}, \boldsymbol{\Sigma}_{k}^{U L}$ can be approximated, under $\beta \ll 1$ and $\kappa \ll 1$, as in (54) at the bottom of the following page. The covariance matrix of $\mathbf{m}_{j}^{D L}$, $\boldsymbol{\Sigma}_{j}^{D L}$ can be defined similarly, i.e. by replacing $\mathbf{H}_{j}^{U L}, \mathbf{V}_{j}^{U L}$, and $\mathbf{H}_{0}$ in (54) with $\mathbf{H}_{k}^{D L}, \mathbf{V}_{j}^{D L}$, and $\mathbf{H}_{k j}^{D U}$, respectively.

The received signals are processed by linear decoders, denoted as $\mathbf{U}_{k}^{U L}=\left[\mathbf{u}_{k, 1}^{U L}, \ldots, \mathbf{u}_{k, d_{k}^{U L}}^{U L}\right] \in \mathbb{C}^{N_{0} \times d_{k}^{U L}}$, and $\mathbf{U}_{j}^{D L}=\left[\mathbf{u}_{j, 1}^{D L}, \ldots, \mathbf{u}_{j, d_{j}^{D L}}^{D L}\right] \in \mathbb{C}^{N_{j} \times d_{j}^{D L}}$ by the BS and the $j$ th DL user, respectively. Therefore the estimate of data streams of the $k$-th UL user at the BS is given as $\hat{\mathbf{s}}_{k}^{U L}=\left(\mathbf{U}_{k}^{U L}\right)^{H} \mathbf{y}_{0}$, and similarly, the estimate of the date stream of the $j$-th DL user is $\hat{\mathbf{s}}_{j}^{D L}=\left(\mathbf{U}_{j}^{D L}\right)^{H} \mathbf{y}_{j}^{D L}$. Using these estimates, the MSE of the $k$ th UL and $j$ th DL user can be written as in (55) and (56), respectively, shown at the bottom of the following page.

The power of the interference resulting from the UL users and BS at the $l$ th PU can be written as

$$
\begin{aligned}
I_{l}^{P U}=\sum_{k=1}^{K} & \operatorname{tr}\left\{\mathbf { G } _ { l k } \left(\mathbf{V}_{k}^{U L}\left(\mathbf{V}_{k}^{U L}\right)^{H}\right.\right. \\
& \left.\left.+\kappa \operatorname{diag}\left(\mathbf{V}_{k}^{U L}\left(\mathbf{V}_{k}^{U L}\right)^{H}\right)\right) \mathbf{G}_{l k}^{H}\right\} \\
+ & \sum_{j=1}^{J} \operatorname{tr}\left\{\mathbf { G } _ { l } \left(\mathbf{V}_{j}^{D L}\left(\mathbf{V}_{j}^{D L}\right)^{H}\right.\right. \\
& \left.\left.+\kappa \operatorname{diag}\left(\mathbf{V}_{j}^{D L}\left(\mathbf{V}_{j}^{D L}\right)^{H}\right)\right) \mathbf{G}_{l}^{H}\right\},
\end{aligned}
$$

where $\mathbf{G}_{l k} \in \mathbb{C}^{N \times M_{k}}\left(\mathbf{G}_{l} \in \mathbb{C}^{N \times M_{0}}\right)$ is the channel between the $l$ th PU and $k$ th UL user (lth PU and the BS). 


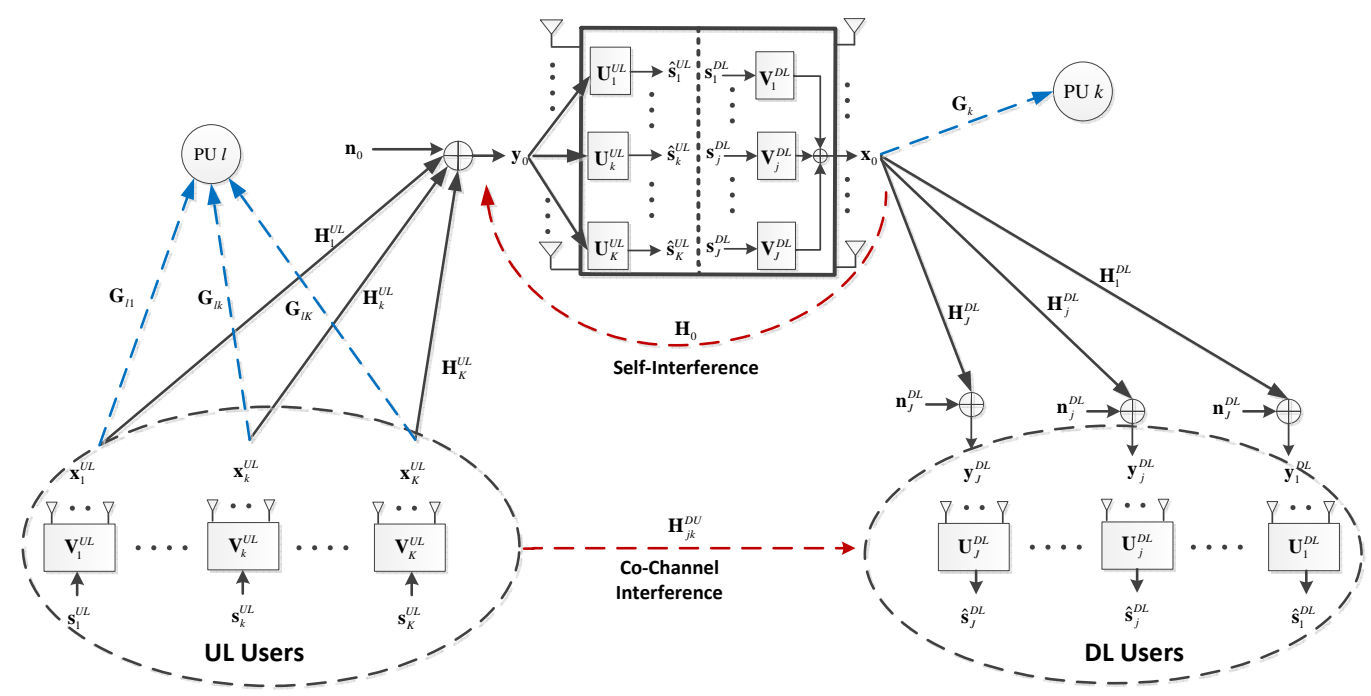

Fig. 2. Full-duplex multi-user MIMO system model.

\section{A. Joint Beamforming Design}

The optimization problem can be formulated as:

$$
\begin{array}{ll}
\min _{\substack{\mathbf{V}_{k}^{U L}, \mathbf{U}_{k}^{U L} \\
\mathbf{V}_{j}^{D L}, \mathbf{U}_{j}^{D L}}} & \sum_{k=1}^{K} \operatorname{tr}\left\{\mathbf{M S E}_{k}^{U L}\right\}+\sum_{j=1}^{J} \operatorname{tr}\left\{\mathbf{M S E}_{j}^{D L}\right\} \\
\text { s.t. } & \operatorname{tr}\left\{\mathbf{V}_{k}^{U L}\left(\mathbf{V}_{k}^{U L}\right)^{H}\right\} \leq P_{k}, k \in \mathcal{S}^{U L}, \\
& \sum_{j=1}^{J} \operatorname{tr}\left\{\mathbf{V}_{j}^{D L}\left(\mathbf{V}_{j}^{D L}\right)^{H}\right\} \leq P_{0} \\
& I_{l}^{P U} \leq \lambda_{l}, l=1, \ldots, L
\end{array}
$$

where $P_{k}$ in (59) is the transmit power constraint at the $k$-th UL user, and $P_{0}$ in (60) is the total power constraint at the BS. We use $\mathcal{S}^{U L}$ and $\mathcal{S}^{D L}$ to represent the set of $K \mathrm{UL}$ and $J$ DL channels, respectively.

1) Simplification of Notations: To simplify the notations, we will combine UL and DL channels, similar to [28].
Denoting $\mathbf{H}_{i j}$ and $\mathbf{n}_{i}$ as

$$
\mathbf{H}_{i j}=\left\{\begin{array}{l}
\mathbf{H}_{j}^{U L}, i \in \mathcal{S}^{U L}, j \in \mathcal{S}^{U L}, \\
\mathbf{H}_{0}, \quad i \in \mathcal{S}^{U L}, j \in \mathcal{S}^{D L}, \mathbf{n}_{i}= \begin{cases}\mathbf{n}_{0}, & i \in \mathcal{S}^{U L}, \\
\mathbf{n}_{i}^{D L}, & i \in \mathcal{S}^{D L}, \\
\mathbf{H}_{i j}^{D U}, i \in \mathcal{S}^{D L}, j \in \mathcal{S}^{U L}, \\
\mathbf{H}_{i}^{D L}, i \in \mathcal{S}^{D L}, j \in \mathcal{S}^{D L},\end{cases}
\end{array}\right.
$$

and referring to $\mathbf{V}_{i}^{X}, \mathbf{U}_{i}^{X}, \boldsymbol{\Sigma}_{i}^{X}, X \in\{U L, D L\}$ as $\mathbf{V}_{i}, \mathbf{U}_{i}$, $\boldsymbol{\Sigma}_{i}$, respectively, the MSE of $i$-th link, $i \in \mathcal{S} \triangleq \mathcal{S}^{U L} \bigcup \mathcal{S}^{D L}$ can be written as

$$
\begin{aligned}
\mathbf{M S E}_{i}= & \left(\mathbf{U}_{i}^{H} \mathbf{H}_{i i} \mathbf{V}_{i}-\mathbf{I}\right)\left(\mathbf{U}_{i}^{H} \mathbf{H}_{i i} \mathbf{V}_{i}-\mathbf{I}\right)^{H} \\
& +\mathbf{U}_{i}^{H} \boldsymbol{\Sigma}_{i} \mathbf{U}_{i},
\end{aligned}
$$

where

$$
\begin{aligned}
\boldsymbol{\Sigma}_{i}= & \sum_{j \in \mathcal{S}, j \neq i} \mathbf{H}_{i j} \mathbf{V}_{j} \mathbf{V}_{j}^{H} \mathbf{H}_{i j}^{H}+\kappa \sum_{j \in \mathcal{S}} \mathbf{H}_{i j} \operatorname{diag}\left(\mathbf{V}_{j} \mathbf{V}_{j}^{H}\right) \mathbf{H}_{i j}^{H} \\
& +\beta \sum_{j \in \mathcal{S}} \operatorname{diag}\left(\mathbf{H}_{i j} \mathbf{V}_{j} \mathbf{V}_{j}^{H} \mathbf{H}_{i j}^{H}\right)+\mathbf{I} .
\end{aligned}
$$

$$
\begin{aligned}
\boldsymbol{\Sigma}_{k}^{U L}= & \sum_{j \neq k}^{K} \mathbf{H}_{j}^{U L} \mathbf{V}_{j}^{U L}\left(\mathbf{V}_{j}^{U L}\right)^{H}\left(\mathbf{H}_{j}^{U L}\right)^{H}+\kappa \sum_{j=1}^{K} \mathbf{H}_{j}^{U L} \operatorname{diag}\left(\mathbf{V}_{j}^{U L}\left(\mathbf{V}_{j}^{U L}\right)^{H}\right)\left(\mathbf{H}_{j}^{U L}\right)^{H} \\
& +\sum_{j=1}^{J} \mathbf{H}_{0}\left(\mathbf{V}_{j}^{D L}\left(\mathbf{V}_{j}^{D L}\right)^{H}+\kappa \operatorname{diag}\left(\mathbf{V}_{j}^{D L}\left(\mathbf{V}_{j}^{D L}\right)^{H}\right)\right) \mathbf{H}_{0}^{H}+\beta \sum_{j=1}^{K} \operatorname{diag}\left(\mathbf{H}_{j}^{U L} \mathbf{V}_{j}^{U L}\left(\mathbf{V}_{j}^{U L}\right)^{H}\left(\mathbf{H}_{j}^{U L}\right)^{H}\right) \\
& +\beta \sum_{j=1}^{J} \operatorname{diag}\left(\mathbf{H}_{0} \mathbf{V}_{j}^{D L}\left(\mathbf{V}_{j}^{D L}\right)^{H} \mathbf{H}_{0}^{H}\right)+\mathbf{I}_{N_{0}} .
\end{aligned}
$$

$$
\begin{aligned}
\mathbf{M S E}_{k}^{U L} & =\left(\left(\mathbf{U}_{k}^{U L}\right)^{H} \mathbf{H}_{k}^{U L} \mathbf{V}_{k}^{U L}-\mathbf{I}_{d_{k}^{U L}}\right)\left(\left(\mathbf{U}_{k}^{U L}\right)^{H} \mathbf{H}_{k}^{U L} \mathbf{V}_{k}^{U L}-\mathbf{I}_{d_{k}^{U L}}\right)^{H}+\left(\mathbf{U}_{k}^{U L}\right)^{H} \boldsymbol{\Sigma}_{k}^{U L} \mathbf{U}_{k}^{U L}, \\
\mathbf{M S E}_{j}^{D L} & =\left(\left(\mathbf{U}_{j}^{D L}\right)^{H} \mathbf{H}_{j}^{D L} \mathbf{V}_{j}^{D L}-\mathbf{I}_{d_{j}^{D L}}\right)\left(\left(\mathbf{U}_{j}^{D L}\right)^{H} \mathbf{H}_{j}^{D L} \mathbf{V}_{j}^{D L}-\mathbf{I}_{d_{j}^{D L}}\right)^{H}+\left(\mathbf{U}_{j}^{D L}\right)^{H} \boldsymbol{\Sigma}_{j}^{D L} \mathbf{U}_{j}^{D L} .
\end{aligned}
$$


2) Transceiver Design: Using the simplified notations, the optimization problem (58)-(61) can be rewritten as

$$
\begin{array}{ll}
\min _{\mathbf{V}_{i}, \mathbf{U}_{i}} & \sum_{i \in \mathcal{S}} \operatorname{tr}\left\{\mathbf{M S E}_{i}\right\} \\
\text { s.t. } & \operatorname{tr}\left\{\mathbf{V}_{i} \mathbf{V}_{i}^{H}\right\} \leq P_{i}, i \in \mathcal{S}^{U L}, \\
& \sum_{i \in \mathcal{S}^{D L}} \operatorname{tr}\left\{\mathbf{V}_{i} \mathbf{V}_{i}^{H}\right\} \leq P_{0}, \\
& I_{l}^{P U} \leq \lambda_{l}, l=1, \ldots, L .
\end{array}
$$

The optimization problem (64)-(67) has the same formulation as the optimization problems proposed for MIMO interference channels, and thus under the fixed receive beamforming matrices, we can apply the individual-power constrained transceiver design proposed in Section III for UL users, $i \in \mathcal{S}^{U L}$, and apply the sum-power constrained transceiver design proposed in Section IV-B for DL users, $i \in \mathcal{S}^{D L}$.

\section{Simulation Results}

In this section, we numerically investigate the MSE-based optimization problems for FD MIMO interference channel and cellular systems as a function of signal-to-noise ratio (SNR), interference-to-noise ratio (INR), and dynamic range parameters $\kappa$ and $\beta$. The tolerance (the difference between MSE of two iterations) of the proposed iterative algorithm is set to $10^{-4}$, the maximum number of iterations is set to 100 , and the results are averaged over 1000 independent channel realizations. Since the optimization problems we are dealing with are non-convex, we need to choose good initialization points to have a suboptimal solution with a good performance. In this paper, we use right singular matrices initialization [46].

\section{A. Interference Channel}

For FD MIMO interference channel, for brevity, we set the same number of transmit and receive antennas at each node, i.e. $M_{i}=N_{i}=N, i \in \mathcal{K}$, and each transmitter sends same number of data streams $d_{i}=N, i \in \mathcal{K}$. We also set the same transmit power constraint for each node in the system, i.e., $P_{i}^{(b)}=N, \forall(i, b)$. We define SNR of the nodes in the $i$-th pair as $\mathrm{SNR}_{i}=\mathrm{SNR} \triangleq \rho_{i} N$, and the INR from the nodes in the $j$-th pair to the nodes in the $i$-th pair as $\mathrm{INR}_{i j}=$ INR $\triangleq \eta_{i j} N, i \neq j$. The INR of the self-interference channel at the nodes in the $i$-th pair is denoted as INR $_{\mathrm{SI}}$. The cognitive radio system is installed within the service range of a primary network having $L=2$ PUs. For simplicity, we set the same maximum allowed interfering power to the PUs (i.e., $\lambda=$ $\left.\lambda_{l}, l=1, \ldots, L\right)$ and same channel gains $\mu=\mu_{l i}^{(b)}, l=$ $1, \ldots, L, i \in \mathcal{K}, b=1,2$.

Fig. 3 illustrates the convergence behavior of the proposed Sum-MSE and Min-Max algorithms. It shows that the proposed algorithms converge in few steps, and it does so monotonically.

In our next example, we examine the sum-rate performance of the proposed Sum-MSE algorithm under individual and sum-power constraints, and Min-Max algorithm. The sum-rate

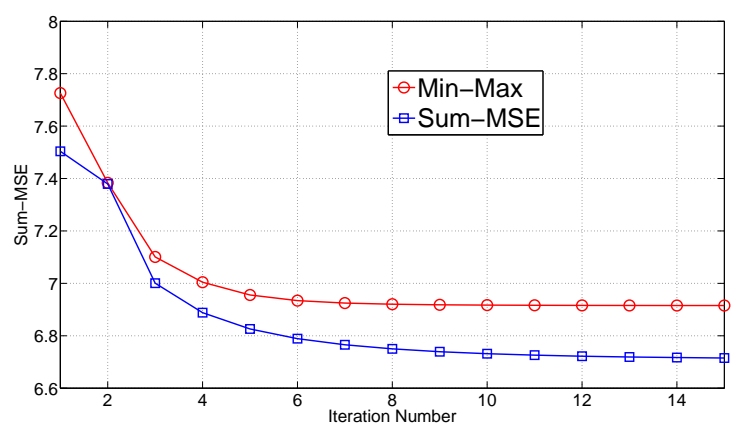

Fig. 3. Convergence behavior of Sum-MSE and Min-Max algorithms. Here, $N=2, K=3, \kappa=\beta=-40 \mathrm{~dB}, \mathrm{SNR}=20 \mathrm{~dB}, \mathrm{INR}=10 \mathrm{~dB}$, $\mathrm{INR}_{\mathrm{SI}}=20 \mathrm{~dB}, \mu=0 \mathrm{~dB}, \lambda=0 \mathrm{~dB}$.

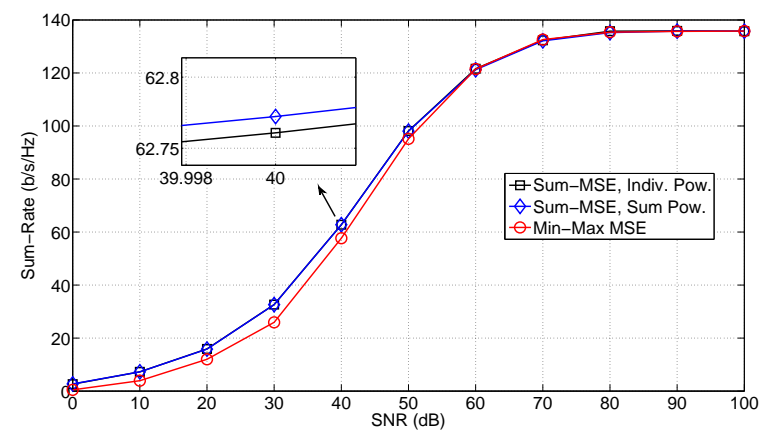

Fig. 4. Sum-rate comparison of the proposed algorithm versus SNR. Here $N=2, K=3, \kappa=\beta=-40 \mathrm{~dB}, \mathrm{INR}=10 \mathrm{~dB}, \mathrm{INR}_{\mathrm{SI}}=20 \mathrm{~dB}$, $\mu=0 \mathrm{~dB}, \lambda=0 \mathrm{~dB}$.

of the MIMO interference channel can be expressed as

$$
I_{\text {sum }}=\sum_{i=1}^{K} \sum_{b=1}^{2} \sum_{k=1}^{d_{i}} \log _{2}\left(1+\operatorname{SINR}_{i_{k}}^{(b)}\right),
$$

where $\operatorname{SINR}_{i_{k}}^{(b)}$ is SINR of the $k$ th stream of node $i^{(b)}$ defined in (69) shown at the bottom of the following page. In (69), $\mathbf{r}_{i_{k}}^{(a)}$ is the $k$ th row of $\mathbf{R}_{i}^{(a)}$, and $\mathbf{v}_{i_{k}}^{(b)}$ is the $k$ th column of $\mathbf{V}_{i}^{(b)}$. As it is seen from Fig. 4, Sum-MSE algorithms achieve higher sum-rate than Min-Max algorithm, since they are designed to achieve the minimum total MSE of all the nodes in the system. Moreover, sum-power constrained and individual power constrained problems perform similar to each other, but sum-power constrained problem achieves higher sum-rate than the individual-power constrained problem, because the sumpower constrained problem is more relaxed than individual power constrained, and thus can allocate more power to the node that contributes more to achieve higher sum-rate.

The next example computes the MSE values for each node in the system for the Sum-MSE and Min-Max schemes out of one channel realization. We can see in Fig. 5 that the Sum-MSE scheme achieves the minimum total MSE over all the nodes and the Min-Max scheme introduces fairness, by ensuring that the all the nodes have almost the same MSE.

In our next example, we investigate the performance of the proposed Sum-MSE minimization algorithm under transmitter/receiver impairments $(\kappa, \beta)$ in Fig. 6. As it is seen in 


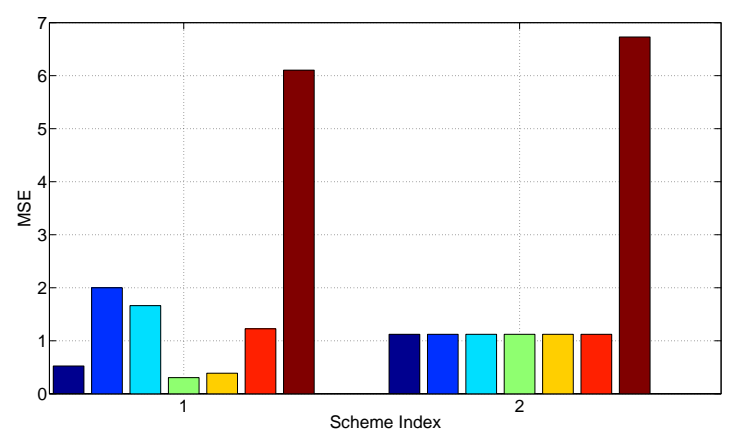

Fig. 5. MSE distribution of Sum-MSE and Min-Max MSE algorithms. The schemes 1 and 2 correspond to Sum-MSE and Min-Max MSE, respectively. For each scheme, the first six bars are the achieved user MSEs and the seventh bar is the sum MSE. Here $N=2, K=3, \kappa=\beta=-40 \mathrm{~dB}, \mathrm{SNR}=20 \mathrm{~dB}$, $\mathrm{INR}=10 \mathrm{~dB}, \mathrm{INR}_{\mathrm{SI}}=20 \mathrm{~dB}, \mu=0 \mathrm{~dB}, \lambda=0 \mathrm{~dB}$.

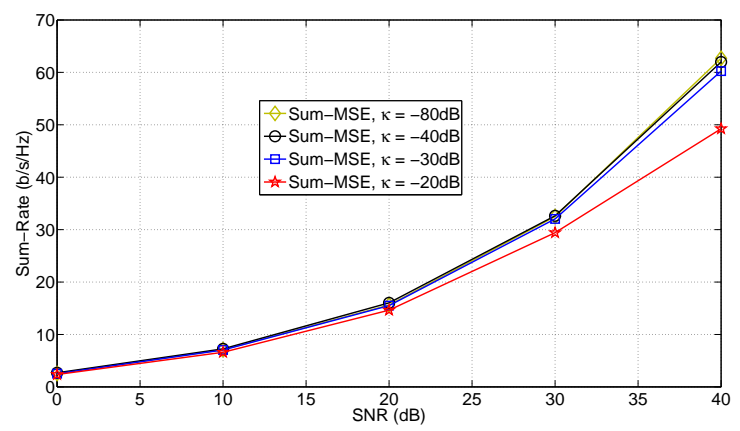

Fig. 6. Sum-rate comparison of the Sum-MSE algorithm with different $\kappa=\beta$ values versus $\mathrm{SNR}$. Here $N=2, K=3, \mathrm{INR}=10 \mathrm{~dB}, \mathrm{INR}_{\mathrm{SI}}=20 \mathrm{~dB}$, $\mu=0 \mathrm{~dB}, \lambda=0 \mathrm{~dB}$.

Fig. 6, at low SNR values, since the thermal noise power dominates the transmitter/receiver distortion power, the sum-rate achieved under different $\kappa, \beta$ are close to each other. But, as the SNR increases, transmitter/receiver distortion power starts dominating the thermal noise power, and the performance of the system is determined by $\kappa$ and $\beta$. Moreover, decreasing $\kappa$ and $\beta$ (decreasing the distortion power) has a diminishing gain on the sum-rate performance of the system.

In the next example, we examine the MSE performance of the proposed Sum-MSE algorithm for various maximum allowed interference at each PU, i.e., $\lambda$. It can be seen from Fig. 7 that as the interference constraint $\lambda$ decreases, the performance gets worse and MSE increases, since a lower interference threshold imposes a more stringent constraint.

\section{B. Cellular System}

In this section, we numerically investigate the MSE-based problem in MIMO FD multi-user system. We compare the

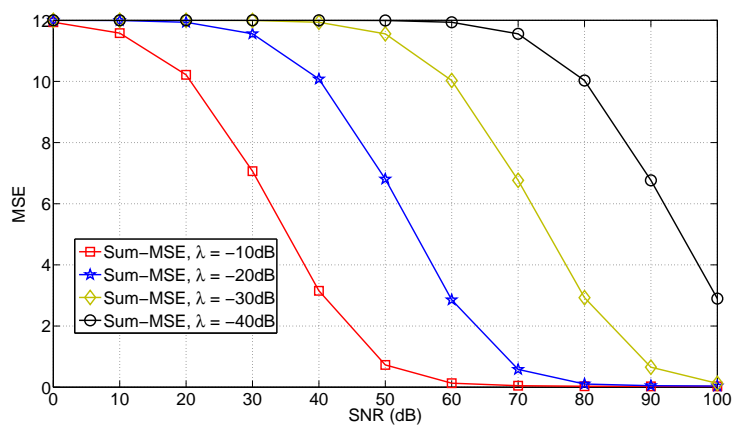

Fig. 7. MSE of cognitive radio system with different $\lambda$ values versus SNR. Here $N=2, K=3, \kappa=\beta=-40 \mathrm{~dB}, \mathrm{INR}=10 \mathrm{~dB}, \mathrm{INR}_{\mathrm{SI}}=20 \mathrm{~dB}$, $\mu=0 \mathrm{~dB}$.

proposed algorithm with the HD algorithm under the 3GPP LTE specifications for small cell deployments [62]. We consider small cells, since they are considered to be suitable for deployment of FD technology due to low transmit powers, short transmission distances and low mobility [2], [3], [63]. A single hexagonal cell having a BS in the center with $M_{0}=2$ transmit and $N_{0}=2$ receive antennas with randomly distributed $K=3 \mathrm{UL}$ and $J=3 \mathrm{DL}$ users equipped with 2 antennas is simulated ${ }^{6}$. The cognitive radio system has $L=2$ PUs, with the same maximum allowed interfering power (i.e., $\lambda_{l}=0 \mathrm{~dB}$ ). The channel between BS and users (both SUs and PUs) are assumed to experience the path loss model for line-of-sight (LOS), and the channel between $\mathrm{UL}$ and DL users are assumed to experience the path loss model for non-line-of-sight (NLOS) communications. Detailed simulation parameters are shown in Table II.

The channel gain between the BS to $k$ th UL user is given by $\mathbf{H}_{k}^{U L}=\sqrt{\kappa_{k}^{U L}} \tilde{\mathbf{H}}_{k}^{U L}$, where $\tilde{\mathbf{H}}_{k}^{U L}$ denotes the small scale fading following a complex Gaussian distribution with zero mean and unit variance, and $\kappa_{k}^{U L}=10^{(-\mathrm{X} / 10)}, X \in\{\mathrm{LOS}, \mathrm{NLOS}\}$ represents the large scale fading consisting of path loss and shadowing, where LOS and NLOS are calculated from a specific path loss model given in Table II. The channels between BS and DL users, between UL users and DL users, between BS and PUs, and between UL users and PUs are defined similarly. For the self-interference channel, we adopt the model in [2] and [13], in which the self-interference channel is distributed as $\mathbf{H}_{0} \sim \mathcal{C N}\left(\sqrt{\frac{C_{\mathrm{SI}}^{2} K_{R}}{1+K_{R}}} \tilde{\mathbf{H}}_{0}, \frac{C_{\mathrm{SI}}^{2}}{1+K_{R}} \mathbf{I}_{N_{0}} \otimes \mathbf{I}_{M_{0}}\right)$, where $K_{R}$ is the Rician factor, $\tilde{\mathbf{H}}_{0}$ is a deterministic matrix, and $C_{\mathrm{SI}}^{2}$

${ }^{6}$ Note that although the BS has $N_{0}+M_{0}$ antennas in total, similar to [28], we assume that only $M_{0}\left(N_{0}\right)$ antennas can be used for transmission (reception) in HD mode. The reason is that in practical systems RF frontends are scarce resources, since they are much more expensive than antennas. Therefore, we assume that BS only has $M_{0}$ transmission front-ends and $N_{0}$ receiving front-ends, and do not carry out antenna partitioning.

$$
\operatorname{SINR}_{i_{k}}^{(b)}=\frac{\rho_{i} \mathbf{r}_{i_{k}}^{(a)} \mathbf{H}_{i i}^{(a b)} \mathbf{v}_{i_{k}}^{(b)}\left(\mathbf{v}_{i_{k}}^{(b)}\right)^{H}\left(\mathbf{H}_{i i}^{(a b)}\right)^{H}\left(\mathbf{r}_{i_{k}}^{(a)}\right)^{H}}{\mathbf{r}_{i_{k}}^{(a)}\left(\boldsymbol{\Sigma}_{i}^{(a)}+\sum_{j \neq k} \rho_{i} \mathbf{H}_{i i}^{(a b)} \mathbf{v}_{i_{j}}^{(b)}\left(\mathbf{v}_{i_{j}}^{(b)}\right)^{H}\left(\mathbf{H}_{i i}^{(a b)}\right)^{H}\right)\left(\mathbf{r}_{i_{k}}^{(a)}\right)^{H}}
$$


TABLE II

Simulation PARAMETERS

\begin{tabular}{|l|l|}
\hline Parameter & Settings \\
\hline Cell Radius & $40 \mathrm{~m}$ \\
\hline Carrier Frequency & $2 \mathrm{GHz}$ \\
\hline Bandwidth & $10 \mathrm{MHz}$ \\
\hline Thermal Noise Density & $-174 \mathrm{dBm} / \mathrm{Hz}$ \\
\hline Noise Figure & BS: $13 \mathrm{~dB}$, User: $9 \mathrm{~dB}$ \\
\hline $\begin{array}{l}\text { Path Loss }(\mathrm{dB}) \text { between BS and users } \\
(d \text { in km })\end{array}$ & $103.8+20.9 \log _{10} d$ \\
\hline Path Loss $(\mathrm{dB})$ between users $(d$ in $\mathrm{km})$ & $145.4+37.5 \log _{10} d$ \\
\hline Shadowing Standard Deviation & LOS: $3 \mathrm{~dB}, \mathrm{NLOS}: 4 \mathrm{~dB}$ \\
\hline
\end{tabular}

denotes the self-interference attenuation level [2] $]^{7}$.

The average sum-rate achieved in FD system for uplink (FD-UL), downlink (FD-DL), and the entire system (FDSum) is shown in Fig. 8. It is seen that while the sum-rate achieved in FD-UL system always decreases as $C_{\mathrm{SI}}^{2}$ increases, the sum-rate achieved in FD-DL system decreases until a certain value of $C_{\mathrm{SI}}^{2}=-80 \mathrm{~dB}$ and increases after that. The decrease in the sum-rate performance of the FD-UL system is intuitive, since as the self-interference suppression capability decreases, a greater amount of self-interference power is added to the background thermal noise. The changing sum-rate performance of the FD-DL system is explained as follows. Since the proposed algorithm optimizes the UL and DL channels jointly, i.e. Sum-MSE minimization of the entire FD system, at low $C_{\mathrm{SI}}^{2}$ values, the joint optimization scheme slightly reduces the transmit power of the DL channel to maintain a good performance of UL system. But, as $C_{\mathrm{SI}}^{2}$ increases, the selfinterference power starts overwhelming the desired signals coming from the UL users, which reduces the achievable sum-rate in the UL channel. Thus, the performance of the entire system is determined mostly by the DL transmission. Therefore, reducing the transmit power in the UL channel and concentrating on DL channel is more beneficial. And also, as the transmission power of the UL users is reduced, CCI is also reduced, resulting in improved performance in the DL channel $^{8}$. The same observation for sum-rate maximization problem has been reported in [2].

Moreover, the sum-rate comparison of FD and HD systems in UL and DL channels is also depicted in Fig. 8. As it is seen, the sum-rate achieved in FD-DL system is always higher than that of HD-DL system, while FD-UL outperforms HD-UL in terms of sum-rate only when the self-interference

\footnotetext{
${ }^{7}$ Similar to [2], without loss of generality, we set $K_{R}=1$ and $\tilde{\mathbf{H}}_{0}$ to be the matrix of all ones for all experiments.

${ }^{8}$ Note that as seen in Fig. 8, at very high self-interference cancellation levels, the DL rate is lower than the UL rate. The reason is that the quality of the DL channel is degraded by the CCI, while the UL channel is not affected by the self-interference at high self-interference cancellation levels. Since the DL service is larger than the UL service in general, in order to give priority to (increase) the DL rate, we can take weighted sum-MSE as the objective function, and give DL larger weights. The weighted sum-MSE problem does not change the proposed algorithm.
}

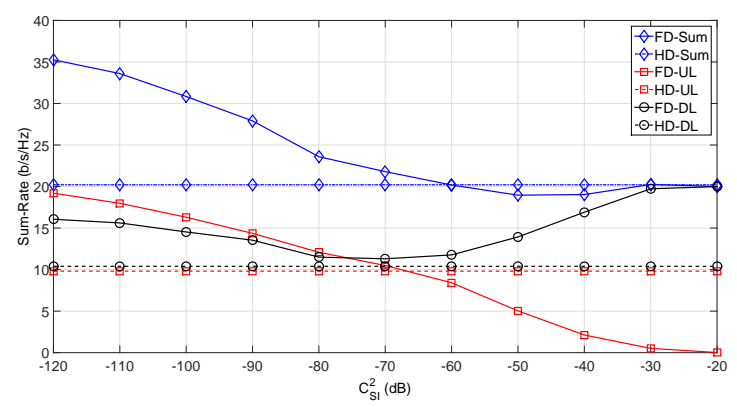

Fig. 8. Sum-rate achieved in full-duplex cellular system versus $C_{\mathrm{SI}}^{2}(\mathrm{~dB})$. Here $\kappa=\beta=-40 \mathrm{~dB}$.

is substantially suppressed ${ }^{9}$. The sum-rate gains of the FD system over HD system as a function of $C_{\mathrm{SI}}^{2}$ is shown in Table III. It is demonstrated that for FD system to achieve a higher sum-rate than $\mathrm{HD}$ system, $C_{\mathrm{SI}}^{2}$ must be at least $-70 \mathrm{~dB}$.

\section{CONCLUSION}

In this work, we have studied the MSE-based transceiver design problems for a FD MIMO cognitive interference channel that suffers from self-interference and inter-user interference under the limited DR at the transmitters and receivers. Since the globally optimal solution is difficult to obtain due to the non-convex nature of the problems, an alternating algorithm that iterates between transmit and receiving beamforming matrices while keeping the other fixed is proposed. It is shown in the simulations that the proposed Sum-MSE minimization scheme achieves the minimum total MSE, and the proposed Min-Max scheme almost achieves the same MSE for every user. Therefore, the Sum-MSE minimization algorithm can be used when there are only best effort services in the system, while it is better to use Min-Max MSE algorithm when there are services with QoS requirements because of the fairness it introduces. Moreover, we show that the proposed algorithm is not only applicable to FD MIMO cognitive interference channels, but also applicable to FD cognitive cellular systems. It has been shown in simulations that the sum-rate achieved by FD system is higher than that of HD system under reasonable self-interference cancellation values.

\section{APPENDIX}

Using (12) and (14), $\mathrm{MSE}_{i}=\operatorname{tr}\left\{\mathbf{M S E}_{i}\right\}$ can be written as in (70) given at the bottom of the following page. Applying the $\operatorname{vec}(\cdot)$ operation, and the identity $\|\operatorname{vec}(\mathbf{A})\|_{2}^{2}=\operatorname{tr}\left\{\mathbf{A} \mathbf{A}^{H}\right\}$, $\mathrm{MSE}_{i}$ in (70) can be rewritten as in (71) given at the bottom of the following page. Then using the identity $\operatorname{vec}(\mathbf{A B C})=$ $\left(\mathbf{C}^{T} \otimes \mathbf{A}\right) \operatorname{vec}(\mathbf{B}),(71)$ can be written as (25).

\footnotetext{
${ }^{9}$ If the residual self-interference is high, it is concluded that FD mode is not a feasible choice. If the level of residual self-interference is time varying and can be measured in real time, then this paper also suggests that a dynamic switching between FD and HD modes may have an advantage. By using a multiple time-slot data transmission as in [17], [18], we can exploit both spatial and temporal freedoms of the MIMO links. Particularly, the use of distinct time slots gives the freedom to switch between FD and HD signaling depending on the power of the self-interference channel.
} 
TABLE III

AVERAGE RATE GAIN OF FULL-DUPLEX UPLINK (DOWNLINK) SYSTEM OVER HALF-DUPLEX UPLINK (DOWNLINK) SYSTEM

\begin{tabular}{|l|l|l|l|l|l|l|l|l|l|l|}
\hline$C_{\text {SI }}^{2}$ & $-110 \mathrm{~dB}$ & $-100 \mathrm{~dB}$ & $-90 \mathrm{~dB}$ & $-80 \mathrm{~dB}$ & $-70 \mathrm{~dB}$ & $-60 \mathrm{~dB}$ & $-50 \mathrm{~dB}$ & $-40 \mathrm{~dB}$ & $-30 \mathrm{~dB}$ & $-20 \mathrm{~dB}$ \\
\hline Uplink & $83 \%$ & $65 \%$ & $45 \%$ & $23 \%$ & $3 \%$ & $-16 \%$ & $-51 \%$ & $-78 \%$ & $-94 \%$ & $-99 \%$ \\
\hline Downlink & $50 \%$ & $40 \%$ & $25 \%$ & $12 \%$ & $5 \%$ & $14 \%$ & $36 \%$ & $65 \%$ & $91 \%$ & $93 \%$ \\
\hline
\end{tabular}

Similar to (71), $I_{l}^{P U}$ can be written as

$$
\begin{aligned}
I_{l}^{P U}= & \sum_{i=1}^{K} \sum_{b=1}^{2} \mu_{l i}^{(b)}\left(\left\|\operatorname{vec}\left(\mathbf{G}_{l i}^{(b)} \mathbf{V}_{i}^{(b)}\right)\right\|_{2}^{2}\right. \\
& \left.+\kappa\left\|\operatorname{vec}\left(\left(\operatorname{diag}\left(\left(\mathbf{G}_{l i}^{(b)}\right)^{H} \mathbf{G}_{l i}^{(b)}\right)\right)^{1 / 2} \mathbf{V}_{i}^{(b)}\right)\right\|_{2}^{2}\right) .
\end{aligned}
$$

$$
\begin{aligned}
\mathrm{MSE}_{i}= & \operatorname{tr}\left\{\mathbf{M S E}_{i}\right\} \\
= & \operatorname{tr}\left\{\left(\sqrt{\rho_{i}} \mathbf{R}_{i}^{(a)} \mathbf{H}_{i i}^{(a b)} \mathbf{V}_{i}^{(b)}-\mathbf{I}_{d_{i}}\right)\left(\sqrt{\rho_{i}} \mathbf{R}_{i}^{(a)} \mathbf{H}_{i i}^{(a b)} \mathbf{V}_{i}^{(b)}-\mathbf{I}_{d_{i}}\right)^{H}\right\} \\
& +\rho_{i} \kappa \operatorname{tr}\left\{\mathbf{R}_{i}^{(a)} \mathbf{H}_{i i}^{(a b)} \operatorname{diag}\left(\mathbf{V}_{i}^{(b)}\left(\mathbf{V}_{i}^{(b)}\right)^{H}\right)\left(\mathbf{H}_{i i}^{(a b)}\right)^{H}\left(\mathbf{R}_{i}^{(a)}\right)^{H}\right\} \\
& +\beta \rho_{i} \operatorname{tr}\left\{\mathbf{R}_{i}^{(a)} \operatorname{diag}\left(\mathbf{H}_{i i}^{(a b)} \mathbf{V}_{i}^{(b)}\left(\mathbf{V}_{i}^{(b)}\right)^{H}\left(\mathbf{H}_{i i}^{(a b)}\right)^{H}\right)\left(\mathbf{R}_{i}^{(a)}\right)^{H}\right\} \\
& +\eta_{i i} \kappa \operatorname{tr}\left\{\mathbf{R}_{i}^{(a)} \mathbf{H}_{i i}^{(a a)} \operatorname{diag}\left(\mathbf{V}_{i}^{(a)}\left(\mathbf{V}_{i}^{(a)}\right)^{H}\right)\left(\mathbf{H}_{i i}^{(a a)}\right)^{H}\left(\mathbf{R}_{i}^{(a)}\right)^{H}\right\} \\
& +\beta \eta_{i i} \operatorname{tr}\left\{\mathbf{R}_{i}^{(a)} \operatorname{diag}\left(\mathbf{H}_{i i}^{(a a)} \mathbf{V}_{i}^{(a)}\left(\mathbf{V}_{i}^{(a)}\right)^{H}\left(\mathbf{H}_{i i}^{(a a)}\right)^{H}\right)\left(\mathbf{R}_{i}^{(a)}\right)^{H}\right\} \\
& +\sum_{j=1, j \neq i}^{K} \sum_{c=1}^{2} \eta_{i j} \operatorname{tr}\left\{\mathbf{R}_{i}^{(a)} \mathbf{H}_{i j}^{(a c)} \mathbf{V}_{j}^{(c)}\left(\mathbf{V}_{j}^{(c)}\right)^{H}\left(\mathbf{H}_{i j}^{(a c)}\right)^{H}\left(\mathbf{R}_{i}^{(a)}\right)^{H}\right\} \\
& +\sum_{j=1, j \neq i}^{K} \sum_{c=1}^{2} \eta_{i j} \kappa \operatorname{tr}\left\{\mathbf{R}_{i}^{(a)} \mathbf{H}_{i j}^{(a c)} \operatorname{diag}\left(\mathbf{V}_{j}^{(c)}\left(\mathbf{V}_{j}^{(c)}\right)^{H}\right)\left(\mathbf{H}_{i j}^{(a c)}\right)^{H}\left(\mathbf{R}_{i}^{(a)}\right)^{H}\right\} \\
& +\sum_{j=1, j \neq i}^{K} \sum_{c=1}^{2} \eta_{i j} \beta \operatorname{tr}\left\{\mathbf{R}_{i}^{(a)} \operatorname{diag}\left(\mathbf{H}_{i j}^{(a c)} \mathbf{V}_{j}^{(c)}\left(\mathbf{V}_{j}^{(c)}\right)^{H}\left(\mathbf{H}_{i j}^{(a c)}\right)^{H}\right)\left(\mathbf{R}_{i}^{(a)}\right)^{H}\right\}+\operatorname{tr}\left\{\mathbf{R}_{i}^{(a)}\left(\mathbf{R}_{i}^{(a)}\right)^{H}\right\} .
\end{aligned}
$$

$$
\begin{aligned}
& \operatorname{MSE}_{i}=\left\|\operatorname{vec}\left(\sqrt{\rho_{i}} \mathbf{R}_{i}^{(a)} \mathbf{H}_{i i}^{(a b)} \mathbf{V}_{i}^{(b)}\right)-\operatorname{vec}\left(\mathbf{I}_{d_{i}}\right)\right\|_{2}^{2}+\rho_{i} \beta\left\|\operatorname{vec}\left(\left(\operatorname{diag}\left(\left(\mathbf{R}_{i}^{(a)}\right)^{H} \mathbf{R}_{i}^{(a)}\right)\right)^{1 / 2} \mathbf{H}_{i i}^{(a b)} \mathbf{V}_{i}^{(b)}\right)\right\|_{2}^{2} \\
& +\rho_{i} \kappa\left\|\operatorname{vec}\left(\left(\operatorname{diag}\left(\left(\mathbf{H}_{i i}^{(a b)}\right)^{H}\left(\mathbf{R}_{i}^{(a)}\right)^{H} \mathbf{R}_{i}^{(a)} \mathbf{H}_{i i}^{(a b)}\right)\right)^{1 / 2} \mathbf{V}_{i}^{(b)}\right)\right\|_{2}^{2} \\
& +\eta_{i i} \kappa\left\|\operatorname{vec}\left(\left(\operatorname{diag}\left(\left(\mathbf{H}_{i i}^{(a a)}\right)^{H}\left(\mathbf{R}_{i}^{(a)}\right)^{H} \mathbf{R}_{i}^{(a)} \mathbf{H}_{i i}^{(a a)}\right)\right)^{1 / 2} \mathbf{V}_{i}^{(a)}\right)\right\|_{2}^{2} \\
& +\sum_{j=1, j \neq i}^{K} \sum_{c=1}^{2} \eta_{i j}\left\|\operatorname{vec}\left(\mathbf{R}_{i}^{(a)} \mathbf{H}_{i j}^{(a c)} \mathbf{V}_{j}^{(c)}\right)\right\|_{2}^{2}+\eta_{i i} \beta\left\|\operatorname{vec}\left(\left(\operatorname{diag}\left(\left(\mathbf{R}_{i}^{(a)}\right)^{H} \mathbf{R}_{i}^{(a)}\right)\right)^{1 / 2} \mathbf{H}_{i i}^{(a a)} \mathbf{V}_{i}^{(a)}\right)\right\|_{2}^{2} \\
& +\sum_{j=1, j \neq i}^{K} \sum_{c=1}^{2} \eta_{i j} \kappa\left\|\operatorname{vec}\left(\left(\operatorname{diag}\left(\left(\mathbf{H}_{i j}^{(a c)}\right)^{H}\left(\mathbf{R}_{i}^{(a)}\right)^{H} \mathbf{R}_{i}^{(a)} \mathbf{H}_{i j}^{(a c)}\right)\right)^{1 / 2} \mathbf{V}_{j}^{(c)}\right)\right\|_{2}^{2} \\
& +\sum_{j=1, j \neq i}^{K} \sum_{c=1}^{2} \eta_{i j} \beta\left\|\operatorname{vec}\left(\left(\operatorname{diag}\left(\left(\mathbf{R}_{i}^{(a)}\right)^{H} \mathbf{R}_{i}^{(a)}\right)\right)^{1 / 2} \mathbf{H}_{i j}^{(a c)} \mathbf{V}_{j}^{(c)}\right)\right\|_{2}^{2}+\operatorname{tr}\left\{\mathbf{R}_{i}^{(a)}\left(\mathbf{R}_{i}^{(a)}\right)^{H}\right\} .
\end{aligned}
$$


interference cancellation," IEEE Trans. Wireless Commun., vol. 8, no. 8, pp. 3933-3938, Aug. 2009.

[2] D. Nguyen, L. Tran, P. Pirinen, and M. Latva-aho, "On the spectral efficiency of full-duplex small cell wireless systems," IEEE Trans. Wireless Commun., vol. 13, no. 9, pp. 4896-4910, Sept. 2014.

[3] S. Goyal, P. Liu, S. Panwar, R. DiFazio, R. Yang, J. Li, and E. Bala, "Improving small cell capacity with common-carrier full duplex radios," in Proc. IEEE Int. Conf. Commun. (ICC 2014), pp. 4987-4993, June 2014.

[4] A. C. Cirik, K. Rikkinen, Y. Rong, and T. Ratnarajah, "A subcarrier and power allocation algorithm for OFDMA full-duplex systems," accepted to IEEE European Conf. Networks and Commun. (EuCNC), June. 2015.

[5] A. C. Cirik, K. Rikkinen, and M. Latva-aho, "Joint subcarrier and power allocation for sum-rate maximization in OFDMA full-duplex systems," accepted to IEEE Vehicular Tech. Conf. (VTC2015-Spring), May., 2015.

[6] W. Afifi and M. Krunz, "Exploiting self-interference suppression for improved spectrum awareness/efficiency in cognitive radio systems." in Proc. of the IEEE INFOCOM'13 Conf., pp. 1258-1266, April 2013.

[7] W. Cheng, X. Zhang, and H. Zhang, "Imperfect full duplex spectrum sensing in cognitive radio networks," in Proc. of the 3rd ACM workshop on Cognitive radio networks, pp. 1-6, Sep. 2011.

[8] N. Singh, D. Gunawardena, A. Proutiere, B. Radunovic, H. V. Balan, and P. B. Key, "Efficient and fair MAC for wireless networks with selfinterference cancellation," in WiOpt, pp. 94-101, 2011.

[9] M. Jain, J. I. Choi, T. Kim, D. Bharadia, K. Srinivasan, S. Seth, P. Levis, S. Katti, and P. Sinha, "Practical, real-time, full duplex wireless," in Proc. Mobicom, pp. 301-312, 2011.

[10] T. Riihonen, S. Werner, and R. Wichman, "Mitigation of loopback selfinterference in full-duplex MIMO relays," IEEE Trans. Signal Process., vol. 59, no. 12, pp. 5983-5993, Dec. 2011.

[11] Y. Hua, "An overview of beamforming and power allocation for MIMO relays," in Proc. IEEE Military Commun. Conf., pp. 375-380, Nov. 2010.

[12] M. Vehkapera, T. Riihonen, and R. Wichman, "Asymptotic analysis of full-duplex bidirectional MIMO link with transmitter noise," IEEE Int. Symp. Pers. Indoor and Mob. Radio Commun., pp. 1265-1270, Sep. 2013.

[13] M. Duarte, C. Dick, and A. Sabharwal, "Experiment-driven characterization of full-duplex wireless systems," IEEE Trans. Wireless Commun., vol. 11, no. 12, pp. 4296-4307, Dec. 2012.

[14] Y. Hua, P. Liang, Y. Ma, A. C. Cirik and Q. Gao, "A method for broadband full-duplex MIMO radio," IEEE Signal Process. Letters, vol. 19, no. 12, pp. 793-796, Dec 2012.

[15] Y. Hua, Y. Ma, A. Gholian, Y. Li, A. C. Cirik, P. Liang, "Radio selfinterference cancellation by transmit beamforming, all-analog cancellation and blind digital tuning," Elsevier Signal Process., vol. 108, pp. 322-340, 2014.

[16] D. Bharadia and S. Katti, "Full duplex MIMO radios," USENIX NSDI, pp. 359-372, 2014.

[17] B. P. Day, A. R. Margetts, D. W. Bliss, and P. Schniter, "Full-duplex bidirectional MIMO: Achievable rates under limited dynamic range," IEEE Trans. Signal Process., vol. 60, no. 7, pp. 3702-3713, July 2012.

[18] A. C. Cirik, Y. Rong, and Y. Hua, "Achievable rates of full-duplex MIMO radios in fast fading channels with imperfect channel estimation", IEEE Trans. Signal Processing, vol. 62, pp. 3874-3886, Aug. 2014.

[19] S. P. Herath, and T. Le-Ngoc, "Sum-rate performance and impact of self-interference cancellation on full-duplex wireless systems," IEEE Int. Symp. Pers. Indoor and Mobile Radio Commun. (PIMRC), pp. 881-885, Sept. 2013.

[20] S. Huberman, and T. Le-Ngoc, "Sequential convex programming for full-duplex single-user MIMO systems," IEEE Int. Conf. on Commun. (ICC), pp. 5078-5082, June 2014.

[21] W. Li, J. Lilleberg, and K. Rikkinen, "On rate region analysis of half- and full-duplex OFDM communication links," IEEE J. Sel. Areas Commun., vol. 32, no. 9, pp. 1688-1698, Sept. 2014.

[22] A. C. Cirik, R. Wang, and Y. Hua, "Weighted-sum-rate maximization for bi-directional full-duplex MIMO systems," in Proc. 2013 Asilomar Conf. Signals, Syst. Computers, pp. 1632-1636, Nov. 2013.

[23] A. C. Cirik, R. Wang, Y. Rong, and Y. Hua, "MSE based transceiver designs for bi-directional full-duplex MIMO systems," in Proc. 15th IEEE Workshop on Signal Process. Advances in Wireless Commun. (SPAWC'2014), pp. 384-388, June 2014

[24] J. Zhang, O. Taghizadeh, and M. Haardt, "Transmit strategies for fullduplex point-to-point systems with residual self-interference," in Proc. Int. ITG Workshop on Smart Antennas (WSA 2013), pp. 1-8, Mar. 2013.

[25] T. M. Kim, H. J. Yang, and A. Paulraj, "Distributed sum-rate optimization for full-duplex MIMO system under limited dynamic range," IEEE Signal Process. Letters, vol. 20, no. 6, pp. 555-558, June 2013.
[26] M. Zhou, H. Cui, L. Song, and B. Jiao, "Transmit-receive antenna pair selection in full duplex systems," IEEE Wireless Commun. Letters, vol. 3, no. 1, pp. 34-37, February 2014.

[27] D. Nguyen, L.-N. Tran, P. Pirinen, and M. Latva-aho, "Precoding for full duplex multiuser MIMO systems: Spectral and energy efficiency maximization," IEEE Trans. Signal Process., vol. 61, no. 16, pp. 40384050, Aug. 2013.

[28] S. Li, R. Murch, and V. Lau, "Linear transceiver design for full-duplex multi-user MIMO system," IEEE Int. Conf. Commun. (ICC), pp. 49214926, June 2014.

[29] A. C. Cirik, R. Wang, Y. Hua, and M. Latva-aho "Weighted sum-rate maximization for full-duplex MIMO interference channels," IEEE Trans. Commun., vol. 63, no. 3, pp. 801-815, March. 2015.

[30] S. Haykin, "Cognitive radio: brain-empowered wireless communications," IEEE J. Sel. Areas Commun., vol. 23, no. 2, pp. 201-220, Feb. 2005.

[31] Q. Zhao and B. M. Sadler, "A survey of dynamic spectrum access," IEEE Signal Process. Mag., vol. 24, no. 3, pp. 79-89, May 2007.

[32] G. Scutari, D. P. Palomar, and S. Barbarossa, "Cognitive MIMO radio," IEEE Signal Process. Mag., vol. 25, no. 6, pp. 46-59, Nov. 2008.

[33] G. Scutari, D. Palomar, F. Facchinei, and J.-S. Pang, "Flexible design of cognitive radio wireless systems: From game theory to variational inequality theory," IEEE Signal Proc. Mag., vol. 26, no. 5, pp. 107-123, Sept. 2009.

[34] G. Scutari and D. P. Palomar, "MIMO cognitive radio: A gametheoretical approach," IEEE Trans. Signal Process., vol. 58, no. 2, pp. 761-780, Feb. 2010.

[35] S.-J. Kim and G. B. Giannakis, "Optimal resource allocation for MIMO ad hoc cognitive radio networks," IEEE Trans. Inf. Theory, vol. 57, no. 5, pp. 3117-3131, May 2011.

[36] L. Zhang, Y.-C. Liang, Y. Xin, and H. V. Poor, "Robust cognitive beamforming with partial channel state information," IEEE Trans. Wireless Commun., vol. 8, no. 8, pp. 4143-4153, Aug. 2009

[37] J. Wang, G. Scutari, and D. P. Palomar, "Robust MIMO cognitive radio via game theory," IEEE Trans. Signal Process., vol. 59, no. 3, pp. 11831201, Mar. 2011.

[38] K. Phan, S. Vorobyov, N. Sidiropoulos, and C. Tellambura, "Spectrum sharing in wireless networks via QoS-Aware secondary multicast beamforming," IEEE Trans. Signal Process., vol. 57, no. 6, pp. 2323-2335, Jun. 2009.

[39] T. W. Ban, W. Choi, B. C. Jung, and D. K. Sung, "Multi-user diversity in a spectrum sharing system," IEEE Trans. Wireless Commun., vol. 8, no. 1, pp. 102-106, Jan. 2009.

[40] A. Ghasemi and E. S. Sousa, "Fundamental limits of spectrum-sharing in fading environments," IEEE Trans. Wireless Commun., vol. 6, no. 2, pp. 649-658, Feb. 2007.

[41] D. I. Kim, L. B. Le, and E. Hossain, "Joint rate and power allocation for cognitive radios in dynamic spectrum access environment," IEEE Trans. Wireless Commun., vol. 7, no. 12, pp. 5517-5527, Dec. 2008.

[42] Q. Zhao, S. Geirhofer, L. Tong, and B. M. Sadler, "Opportunistic spectrum access via periodic channel sensing," IEEE Trans. Signal Process., vol. 56, no. 2, pp. 785-796, Feb. 2008.

[43] F. Gao, R. Zhang, Y.-C. Liang, and X. Wang, "Multi-antenna cognitive radio systems: Environmental learning and channel training," in Proc. IEEE ICASSP, Apr. 2009, pp. 2329-2332.

[44] E. A. Gharavol, Y.-C. Liang, and K. Mouthaan, "Robust downlink beamforming in multiuser MISO cognitive radio networks with imperfect channel-state information," IEEE Trans. Veh. Technol., vol. 59, no. 6, pp. 2852-2860, Jul. 2010.

[45] J. M. Peha, "Approaches to spectrum sharing," IEEE Commun. Mag., vol. 43 , no. 2 , pp. 10-12, Feb. 2005

[46] C. E. Chen and W. H. Chung, "An iterative minmax per-stream MSE transceiver design for MIMO interference channel," IEEE Wireless Commun. Lett., vol. 1, no. 3, pp. 229-232, Jun. 2012.

[47] F. Negro, S. P. Shenoy, I. Ghauri, and D. T. M. Slock, "On the MIMO interference channel," in Proc. Information Theory and Applications Workshop, pp. 1-9, Jan. 2010.

[48] J. G. D. Forney, "Shannon meets Wiener II: On MMSE estimation in successive decoding schemes," in Proc. Allerton Conf. Commun., Control, and Computing, Monticello, IL, Sep. 2004

[49] D. P. Palomar, J. M. Cioffi, and M. A. Lagunas, "Joint Tx-Rx beamforming design for multicarrier MIMO channels: A unified framework for convex optimization," IEEE Trans. Signal Process., vol. 51, no. 9, pp. 2381-2401, Sept. 2003.

[50] R. Hunger, M. Joham, and W. Utschick, "On the MSE-duality of the broadcast channel and the multiple access channel," IEEE Trans. Signal Process., vol. 57, no. 2, pp. 698-713, Feb. 2009. 
[51] Y. Rong, X. Tang, and Y. Hua, "A unified framework for optimizing linear nonregenerative multicarrier MIMO relay communication systems," IEEE Trans. Signal Process., vol. 57, no. 12, pp. 4837-4851, Dec. 2009.

[52] H. Suzuki, T. V. A. Tran, I. B. Collings, G. Daniels, and M. Hedley, "Transmitter noise effect on the performance of a MIMO-OFDM hardware implementation achieving improved coverage," IEEE J. Sel. Areas Commun., vol. 26, pp. 867-876, Aug. 2008.

[53] W. Namgoong, "Modeling and analysis of nonlinearities and mismatches in AC-coupled direct-conversion receiver," IEEE Trans. Wireless Commun., vol. 4, pp. 163-173, Jan. 2005.

[54] I. E. Telatar, "Capacity of multi-antenna gaussian channels," Eur. Trans. Telecommun., vol. 10, pp. 585-595, Nov. 1999.

[55] C. Xiao, Y. R. Zheng, and Z. Ding, "Globally optimal linear precoders for finite alphabet signals over complex vector Gaussian channels," IEEE Trans. Signal Process., vol. 59, no. 7, pp. 3301-3314, Jul. 2011.

[56] M. S. Lobo, L. Vandenberghe, S. Boyd, and H. Lebret, "Applications of second order cone programming," Linear Algebra APP., 1998.

[57] J. F. Sturm, "Using SeDuMi 1.02, a MATLAB tool for optimization over symmetric cones," Optim. Methods Softw., vol. 11-12, pp. 625-653, 1999.

[58] Y. Rong and Y. Hua, "Optimal power schedule for distributed MIMO links," IEEE Trans. Wireless Commun., vol. 7, pp. 2896-2900, Aug. 2008.

[59] Y. Rong, Y. Hua, A. Swami, and A. L. Swindlehurst, "Space-time power schedule for distributed MIMO links without instantaneous channel state information at the transmitting nodes," IEEE Trans. Signal Proccess., vol. 56, no. 2, pp. 686-700, Feb. 2008.

[60] Y. Rong and Y. Hua, "Space-time power scheduling of MIMO links Fairness and QoS considerations," IEEE J. Sel. Topics Signal Process., vol. 2, no. 2, pp. 171-180, Apr. 2008.

[61] N. Ul Hassan, C. Yuen, S. Saeed, and Z. Zhang, "Power control for sumrate maximization on interference channels under sum power constraint," IEEE Trans. Vehicular Tech., vol. 64, no. 2, pp. 593-609, Feb. 2015.

[62] 3GPP, TR 36.828, "Further enhancements to LTE time division duplex (TDD) for downlink-uplink (DL-UL) interference management and traffic adaptation (Release 11)," June 2012.

[63] DUPLO project, "System scenarios and technical requirements for fullduplex concept," Deliverable D1.1. [Online]. Available: http://www.fp7duplo.eu/index.php/deliverables.

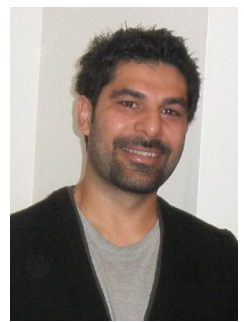

Ali Cagatay Cirik (M'14) received the B.S and M.S. degrees in telecommunications and electronics engineering from Sabanci University, Istanbul, Turkey, in 2007 and 2009, respectively, and Ph.D. degree in electrical engineering from University of California, Riverside in 2014. Currently, he is working as a research scientist at Centre for Wireless Communications, University of Oulu, Finland.

His industry experience includes internships at Mitsubishi Electric Research Labs, Cambridge, MA, in 2012 and at Broadcom Corporation, Irvine, CA, in 2013. His primary research interests are full-duplex communication, MIMO signal processing, and convex optimization.

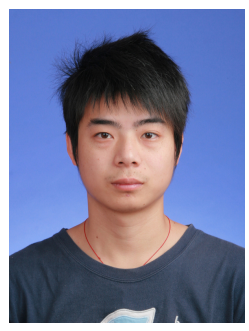

Rui Wang (M'14) received the B.S. degree from Anhui Normal University, Wuhu, China, in 2006, and the M.S. degree from Shanghai University, Shanghai, China, in 2009 and the Ph.D. degree from Shanghai Jiao Tong University, China, in 2013, al in electronic engineering. From Aug. 2012 to Feb. of 2013, he was a visiting Ph.D student at the Department of Electrical Engineering of University of California, Riverside. From Oct. 2013 to Oct. of 2014, he was with the Institute of Network Coding, The Chinese University of Hong Kong as a postdoctoral research associate. Since Oct. 2014, he has been with the College of Electronics and Information Engineering, Tongji University as an assistant professor. His research interests include wireless cooperative communications, MIMO technique, network coding, and OFDM etc.

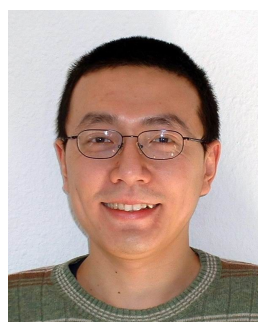

Yue Rong (S'03-M'06-SM'11) received the Ph.D. degree (summa cum laude) in electrical engineering from the Darmstadt University of Technology, Darmstadt, Germany, in 2005.

He was a Post-Doctoral Researcher with the Department of Electrical Engineering, University of California, Riverside, from February 2006 to November 2007. Since December 2007, he has been with the Department of Electrical and Computer Engineering, Curtin University, Bentley, Australia, where he is currently an Associate Professor. His research interests include signal processing for communications, wireless communications, underwater acoustic communications, applications of linear algebra and optimization methods, and statistical and array signal processing. He has published over 120 journal and conference paper in these areas.

Dr. Rong was a recipient of the Best Paper Award at the 2011 International Conference on Wireless Communications and Signal Processing, the Best Paper Award at the 2010 Asia-Pacific Conference on Communications, and the Young Researcher of the Year Award of the Faculty of Science and Engineering at Curtin University in 2010. He is an Associate Editor of theIEEE TRansactions on Signal Processing. He was an Editor of the IEEE WIRELESS COMMUNICATIONS LETTERS from 2012 to 2014, a Guest Editor of the IEEE Journal on SELECTED AREAS IN COMMUNICATIONS special issue on theories and methods for advanced wireless relays, and was a TPC Member for the IEEE ICC, WCSP, IWCMC, and ChinaCom.

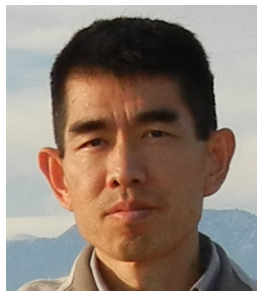

Yingbo Hua (S'86-M'88-SM'92-F'02) received a B.S. degree (1982) from Southeast University, Nanjing, China, and a Ph.D. degree (1988) from Syracuse University, Syracuse, NY. He held a faculty position with the University of Melbourne, Australia, where he was promoted to the rank of Reader and Associate Professor from 1996. He was a visiting professor with Hong Kong University of Science and Technology (1999-2000), and a consultant with Microsoft Research, WA (summer 2000). Since 2001, California at Riverside. he has been a Professor with the University of

Dr. Hua has served as Editor, Guest Editor, Member of Editorial Board and/or Member of Steering Committee for IEEE Transactions on Signal Processing, IEEE Signal Processing Letters, EURASIP Signal Processing, IEEE Signal Processing Magazine, IEEE Journal of Selected Areas in Communications, and IEEE Wireless Communication Letters. He has been a Member of IEEE Signal Processing Society's Technical Committees for Underwater Acoustic Signal Processing, Sensor Array and Multichannel Signal Processing, and Signal Processing for Communication and Networking. $\mathrm{He}$ has served on Technical and/or Organizing Committees for over 50 international conferences and workshops. He has authored/coauthored over 300 articles and coedited three volumes of books, with more than 8000 citations, in the fields of Sensing, Signal Processing and Communications. $\mathrm{He}$ is a Fellow of IEEE and AAAS. 